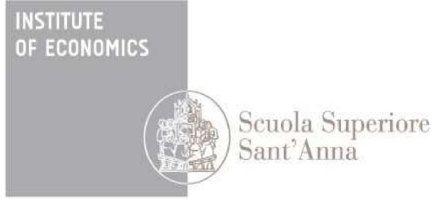

LEM | Laboratory of Economics and Management

Institute of Economics

Scuola Superiore Sant'Anna

Piazza Martiri della Libertà, 33 - 56127 Pisa, Italy ph. +3905088.33 .43$

institute.economics@sssup.it

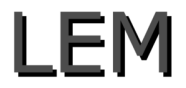

Working Paper Series

\title{
A Dynamical Approach to Conflict Analysis
}

\author{
Sebastian Ille
}

§ Institute of Economics and LEM, Scuola Superiore Sant'Anna, Pisa, Italy 


\title{
A Dynamical Approach to Conflict Analysis
}

\author{
Sebastian Ille*
}

February 10, 2012

\begin{abstract}
The Conflict Analysis approach by Hipel and Fraser (1984) is well equipped to model repeated games. Players are assumed to posses a sequential reasoning that allows them to ( not necessarily correctly) anticipate the reaction of other players to their strategies. An individual's best response strategy is thus defined based on this projection, adding additional stability conditions to strategic choice and increasing the set of potential equilibria beyond pure Nash equilibria. Yet, the original Conflict Analysis approach lacks the ability to genuinely model dynamic repeated games, in which past play defines the condition for future interactions. This article will illustrate how the original model can be adapted to include endogenous individual preferences that are defined by the strategic choice of players during past play, allowing to model the reciprocal connection between preferential change and best response play in repeated games. A dummy game serves as an exemplar and helps to visualise the results obtained from this extension.
\end{abstract}

Keywords: Game Theory, Repeated Games, Computational Methods, Non-Nash Equilibria, Dominated Strategies

JEL classification: C62, C65, D74, D83, D84,

\footnotetext{
*Sant'Anna School of Advanced Studies (Pisa) - Italy. E-Mail: sebastian.ille@ sssup.it
} 


\section{Introduction}

Conflict Analysis is an alternative approach that assumes the capacity of players to extrapolate the reactions of other players to their strategic choices. The sequential reasoning renders it interesting for the analysis and modelisation of repeated games. Ille (2012) has illustrated and critically analysed the underlying assumptions, and obtained results by focusing on a static analysis. In the context of repeated games, the approach presented so far is insufficient to effectively model the dynamics of strategic choice over a sequence of interactions. This article concentrates on the dynamic analysis of games and expands the initial attempt of (Fraser and Hipel, 1984, Ch. 14).

\subsection{The Solution Algorithm}

The basic assumptions of the Conflict Analysis approach are briefly reviewed in the following. For a more detailed explanation refer to Ille (2012). Given an n-person non-cooperative game defined by $G=\left(S_{1}, S_{2}, \ldots, S_{n} ; U_{1}, U_{2}, \ldots, U_{n}\right)$, with player set $N=(1,2,3, \ldots, n)$, and $S_{i}$ being individual $i$ 's strategy set and $S=\times S_{i}$ being the set of strategy profiles. Let there be an individual preference function $U_{i}$ for each $i \in N$ that ranks the strategy profiles according to the individual's preferences over the associated outcomes by assigning all strategy profiles $s=\left(s_{1}, s_{2}, \ldots, s_{n}\right) \in S$ to one of two subsets with respect to any underlying strategy profile $q=\left(\bar{s}_{i}, \bar{s}_{-i}\right)$ !

$$
\begin{aligned}
& p \in U_{i}^{+}(q), \text { iff } O(p) \succ_{i} O(q) \\
& p \in U_{i}^{-}(q), \text { iff } O(p) \preceq_{i} O(q)
\end{aligned}
$$

with $U_{i}^{+}(q) \cap U_{i}^{-}(q)=\emptyset$ and $U_{i}^{+}(q) \cup U_{i}^{-}(q)=S$, and $O(q)$ the outcome associated to $q$. Denote a strategy profiles that can be obtained by a unilateral strategy switch, i.e. a switch of player $i$ given the strategies of all players other than $i$, by $z_{i}(q)=\left(s_{i}, \bar{s}_{-i}\right)$, with any $s_{i} \in S_{i}$. Given the set $Z_{i}(q)$ of all strategy profiles that can be obtained by a unilateral switch of $i$, the set of "dominant profiles" for $q$ is then defined as

$$
\text { DP: } u_{i}^{+}(q)=Z_{i}(q) \cap U_{i}^{+}(q), \forall s_{i} \in S_{i}
$$

In other words, a dominant profile with respect to $q$ is defined by the strategy profile of a unilateral switch of player $i$ to a better response strategy given strategies $\bar{s}_{-i}$ of all players other than $i$.

\footnotetext{
${ }^{1}$ Preferences are assumed to be complete and transitive, though the last assumption is not strictly necessary for the approach, see agin Ille (2012) for details.
} 
Two forms of stability of a player's strategy choice can occur. If a better response strategy is absent, a player is already playing his best response strategy and the underlying strategy profile is termed rationally stable for player $i$.

$$
\text { Rational Stability: } u_{i}^{+}\left(\bar{s}_{i}, \bar{s}_{-i}\right)=\emptyset, \forall s_{i} \in S_{i}
$$

Furthermore, the switch to a better response strategy can entail a probable subsequent switch of another player $j$ to his better response strategy, given the new strategy profile after the switch of player $i$. This may lead to an outcome, not strictly preferred by player $i$ to the outcome defined by the strategy profile from which player $i$ originally switched. Thus, player $i$ refrains from unilaterally choosing this better response strategy. In that case the strategy switch is sequentially sanctioned and the strategy does not qualify as a viable better response strategy. If all better response strategies are sequentially sanctioned, the current strategy is best response and the underlying strategy profile is termed sequentially stable for player $i$. For any player $j$ define $\hat{u}_{j}^{+}(p)=Z_{j}(p) \cap U_{j}^{+}(p)$ as the set of $D P$ s for player $j$ to player $i$ 's dominant profile $p$ for $q$,

$$
\begin{array}{r}
\text { Sequential stability: } \hat{u}_{j}^{+}\left(p=\left(s_{i}^{*}, \bar{s}_{-i}\right)\right) \cap U_{i}^{-}\left(q=\left(\bar{s}_{i}, \bar{s}_{-i}\right)\right) \neq \emptyset \\
\forall s_{i}^{*}: p=\left(s_{i}^{*}, \bar{s}_{-i}\right) \in u_{i}^{+}(q) \text { and for any } j \neq i
\end{array}
$$

If a strategy is neither rational nor sequential stable for player $i$, i.e. neither condition 3 nor condition 4 hold, it is unstable ${ }^{2}$ Define the underlying strategy profile as unstable for player $i$. A strategy profile that consists only of components that are best response strategies, i.e. a strategy profile that is stable for all players, is considered stable, and defines the game's potential equilibrium. Whether the individual strategies in the profile are sequentially or rationally stable, is irrelevant. Notice, however, that only a strategy profile, in which each component is rationally stable, is a Nash equilibrium.

\subsection{Representation}

Since this approach goes beyond the Nash definition of an equilibrium by adding sequential and simultaneous stability, a representation of a game in normal or extensive form is insufficient. It is therefore necessary to spend a few words on the structure of analysis. Each strategy can define a set of actions, such that an individual strategy consisting of $r$ independent actions is defined as $s_{i}=\left(a_{1 i}, a_{2 i}, \ldots, a_{r i}\right)$. A player has the choice whether or not to take a certain action. Define the set $A_{k i}=\left(a_{k i}, \neg a_{k i}\right)$, so set $A_{k i}$ consists of two elements, the first meaning that action $k$ is chosen by player $i$, the second that it is not. Whence we ob-

\footnotetext{
${ }^{2}$ Simultaneous stability is of no interest in this context, as this article will focus solely on repeated games that are played sequentially.
} 
tain that $S_{i} \subseteq \times_{k} A_{k i}$, where the equality holds if none of the actions are mutually exclusive. Hence, each strategy of a player $i$ can be uniquely defined by a binary vector of length equal to the number of actions that player $i$ possesses. Similarly, also each strategy profile can be uniquely identified by a binary vector equal in length to the sum of all available individual actions. Each element in this vector defines an individual action and its value whether the action is chosen or not.

Assume a game with three players, where the strategy set $S_{i}$ is defined by the number of actions $x=\left|\bigcup_{k} A_{k i}\right|$, strategy set $S_{j}$ by the number of actions $y=\left|\bigcup_{k} A_{k j}\right|$, and strategy $S_{h}$ by the number of actions $z=\left|\bigcup_{k} A_{k h}\right|$, implying that the actions are mutually non-exclusive for player $i, j$ and $h$, respectively ${ }^{3}$ In such a three player game, each strategy profile $q=\left(s_{i}, s_{j}, s_{h}\right)$ can be defined by a binary vector of length $x+y+z$, given by $\hat{q}=(\overbrace{I, I, \ldots, I, I}^{x}, \overbrace{I, I, \ldots, I}^{y}, \overbrace{I, I, \ldots, I}^{z})^{T} . I$ denotes a binary value of either 0 or 1 , where 1 implies that the action is chosen, 0 that it is not. Hence, each player individually defines the sequence of this binary vector for a length equal to the number of available actions. As an example, for a three player game, in which each player has two mutually non-exclusive actions, one strategy profile $p$ is defined by $\hat{p}=(0,1,1,0,1,0)^{T}$. The length of this binary vector can be reduced in the case of mutually exclusive actions. If an action $A$ can only be chosen, if an action $B$ is not and the inverse, but one action has to be chosen, then both action can be described by a single digit in the binary vector. $I=1$ could be defined as $A$ is chosen by a player $i$, and thus $I=0$ would mean that $B$ is chosen.

Each such binary vector can be again uniquely defined by a decimal code, calculated as follows: In general the binary vector has $\left|\bigcup_{i \in N}\left(\bigcup_{k} a_{k i}\right)\right|$ digits (less the number of those actions reduced by the aforementioned simplification in the case of mutually exclusive actions) that have either the value 1 or 0 . Like the binary code of a computer this can be rewritten by taking the sum over the products of the digit times two to the power of the position in the vector. Consequently, the example $\hat{p}=(0,1,1,0,1,0)^{T}$ can be written as $0 * 2^{0}+1 * 2^{1}+1 * 2^{2}+0 * 2^{3}+1 * 2^{4}+0 * 2^{5}=22=\dot{p}$. The value of 22 does not represent a preference, but is the short representation of a strategy profile.

A preference order can thus be defined as a vector of length equal to the sum of actions $\left(\left|\bigcup_{i} \bigcup_{k} A_{k i}\right|\right)$, reduced by the actions that are mutually exclusive, that can be transformed into a natural number defining a strategy profile.

Given the assumptions, the preference function $U_{i}$ orders the strategy profiles into the preference vector according to the preferences of player $i$ over the associated outcomes. Since preferences are strictly ordinal, it suffices to note down the natural numbers, identifying each a strategy profile, in a vector, where the position of the component indicates the

\footnotetext{
${ }^{3}|$.$| denotes the cardinality of a set, i.e. absolute number of elements in the set.$
} 
preference. Starting with the most preferred, strategy profiles can be ordered from the left most position to the right. This implies that for strict and transitive preferences each strategy profile can have only one position in the preference vector and it is strictly preferred to all strategy profiles noted further to the right, i.e. for $O(q) \succ_{i} O(p) \rightarrow U_{i}=(\ldots, \dot{q}, \ldots, \dot{p}, \ldots)$.

\section{A fictitious Game of Social Conflict}

In the following I will apply the Conflict Analysis approach to a fictitious game, in which two groups with conflicting interests interact. The game will serve as an exemplar to illustrate how the approach is able to model the interaction dynamics of a repeated game. Beginning with the static analysis of the game that constitutes the basis of the dynamic analysis, the approach is extended step by step to provide a more sophisticated description and analysis of the underlying game. Focus will be placed on the dynamic representation. Starting out with a constant time-homogeneous transition matrix, the matrix is changed as such that it is able to model the transitions between various preference orders. Subsequently, the model is transformed into a more realistic dynamic process, where each state defines the interaction basis for the subsequent state, yet with an exogenously determined transition between preference orders. In a last step, the preference orders are directly determined by the strategy distribution of past play, thus previous states both define subsequent states and the rate of transition between preference orders.

Assume a game with two groups of players $C_{A}$ and $C_{B}$. Further assume that the behaviour of the two conflicting parties can be described by the collective action of all players in each group. This implies that we are not interested in individual decision and the subtle processes inside a group but the aggregate joint action ${ }^{4}$ The game is thus assumed to be sufficiently defined by $\Gamma=\left(S_{A t}, S_{B t} ; U_{A t}, U_{B t}\right)$, where $S_{i t}$ defines the strategy set and $U_{i t}$ the preference order for player group $i$, given $i=A, B$, at time $t$. Suppose both groups have to repeatedly renew a contract (e.g. on the relative monetary pay-off for the joint production of a good, on working conditions or on laws governing the mutual co-existence). Assume that group $B$ is the proposing group that offers a contract and that group $A$ has to decided whether or not to accept. The model is general enough to be interpreted as an abstract representation of a conflict on various social levels, ranging from an interaction between two classic social classes (the working class vs. the capitalists) over the conflict inside a single company (advisory board vs. board of directors) down to the individual level.

\footnotetext{
${ }^{4}$ Notice that this is not equivalent to modelling macroeconomic behaviour on the basis of a representative agent. In this game, no assumptions on the individual preferences and actions exist, i.e. it is not supposed that any action of the group necessarily coincides with individual strategy choice, similar to the negligence of neural processes in the standard economic explanation of individual choices. The model does not start out in a micro level to explain macro dynamics, but assumptions are made on the same level as the general results, thereby evading problems of super- or sub-additivity.
} 
Suppose that $A$ has only a very limited action set $5^{5}$ It can choose whether or not to fulfil $B$ 's contract and whether or not to actively demand a change in the (social) contract (e.g. by revolting or striking). $B$ has a larger action set. First, $B$ decides about the relative share it offers, i.e. how much the contract should benefit itself at the cost of $A$ 's benefit. For simplicity consider this only as the choice between a non-exploitative and an exploitative offer. Second, $B$ considers, whether or not it demands the certification of the terms of the contract, so that group $A$ is legally obliged to fulfil its part of the contract. Third and last, $B$ has to choose whether or not to threaten with drastic additional sanctions, should $A$ not abide to the terms of the contract, in order to pressure $A$ to sign and fulfil the contract. Hence, $A$ can choose between 4 actions (considering that inaction is an action), and $B$ between 6. Consequently, there exist 4 pure strategies for $A$ and 8 pure strategies for $B$ leading to $2^{5}=32$ possible strategy profiles.

To fulfil the contract and to demand a change of the terms is mutually exclusive, as is the inverse. Hence, both the action of fulfilling the contract and of not demanding a change can be represented as a single action, as can be the contrary, resulting in $2^{4}=16$ possible strategy profiles. For notational simplicity the strategy sets can be further reduced by neglecting strategy profiles that are defined by strictly dominated strategies 6 These are the two strategies, in which $B$ does not certify the terms of the contract, but threatens $A$ by sanctions (a literal incredible threat), namely (Exploitation, no Certification, Sanction) and (no Exploitation, no Certification, Sanction). Furthermore, strategies that include the provision of a non-exploitative share of benefit to group A, after it did not accept the contract, are also assumed strictly dominated. This reduces the whole strategy profile set to 9 $(=16-2-2-(4-1))]^{7}$ Each binary representation of a strategy profile (where 1 implies that the action is taken and 0 that it is not) in the strategy set can again be symbolised by a decimal code (see table 1).

The strategy profiles with value 3 and 7 define non-exploitative outcomes, in which both groups have an incentive to abide to a contract that does not advantage one group over another. Strategy profile 0 , in contrast, implies the requirement of a contractual change as none of the groups conforms to the terms of the initial contract 8

If there exists a bijective relation between strategy profile and outcome, an individual's preference over all outcomes determines a unique preference order of the set of strategy profiles. An outcome is, however, not only defined by a strategy profile, but depends on

\footnotetext{
${ }^{5}$ In order to simplify notation I will speak, in the following, of $A$ or $B$, instead of group $A$ or group $B$.

${ }^{6}$ Note, however, that this assumption requires that the strategies are strictly dominated under the assumption of sequential reasoning.

${ }^{7}$ The last assumption of strict dominance excluded $2^{2}$ strategy profiles, of which one has already been excluded by the previous assumption.

${ }^{8} 0$ can be interpreted for example as a period of social revolution or the complete renewal of labour contracts in a company.
} 
Table 1. Strategy profile Set

\begin{tabular}{|c|c|c|c|c|c|c|c|c|c|}
\hline \multicolumn{10}{|l|}{$A$ 's actions: } \\
\hline Abide & 0 & 1 & 1 & 0 & 1 & 1 & 0 & 1 & 1 \\
\hline \multicolumn{10}{|l|}{$B$ 's actions: } \\
\hline No Exploitation & 0 & 0 & 1 & 0 & 0 & 1 & 0 & 0 & 1 \\
\hline Certification & 0 & 0 & 0 & 1 & 1 & 1 & 1 & 1 & 1 \\
\hline Sanction & 0 & 0 & 0 & 0 & 0 & 0 & 1 & 1 & 1 \\
\hline "Decimal code & 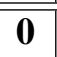 & $\overline{1} \mathbf{1}$ & 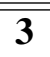 & 4 & 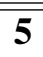 & 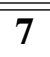 & $\overline{12}$ & 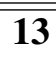 & 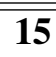 \\
\hline
\end{tabular}

the external circumstances defined by the rules of the game. Further, the preferential evaluation of an outcome is defined by the history of play of the other players that can change the bargaining power, the information and belief a player has (for example on the others bargaining power) and also the evaluation of other players (other-regarding preferences). Thus, assume that an outcome is also defined by the relative bargaining power of a groups and each utility/preference value is affected by the sympathy for the other player group. Further suppose that these change exogenously in time. (This assumption is relaxed later in section 3.2.

According to the value that is assigned to these variables, the strategy profile associated to an outcome and its underlying utility will change in the course of play. So will the preference order. It is unnecessary to explicitly model these variables and sufficient to implicitly incorporate them into the preference vector. Notice, however, that the decimal number does not describe identical outcomes under different assumptions of sympathy and bargaining power. A decimal number represents indeed identical strategy profiles, but outcomes are defined conditional on the value of bargaining power and sympathy. When sympathy of one player group for another player group decreases, strategy profiles that assign relatively higher utility to the latter will offer less utility to the former and rank lower in the preference order. Bargaining power will affect the feasibility and stability of certain actions (e.g. the effect of strikes or the threat of a sanction). A change in bargaining power will thus alter the relative preference for a strategy profile. 9

For the first case assume that initially $A$ prefers above all those strategy profiles, in which $B$ offers a non-exploitative contract. It is indifferent between strategy profile 7 and 3 , and slightly dislikes the threat of sanctions. If $B$ offers an exploitative contract and does not certify the contract, $A$ prefers not to abide to the terms of the contract. If the contract is certified, $A$ prefers the case of no sanction, since this implies lower costs in the case, where

\footnotetext{
${ }^{9}$ Arguably such a model leaves too much room for interpretation of exactly how a change in preference order occurs. Yet, the abstract way, in which complex interactions are analysed, should leave that room of interpretation.
} 
$A$ breaks the agreement. In the second case, $A$ knows that a non-exploitative contract is no longer feasible, since B has appropriated sufficient market power. As a consequence, $A$ loses sympathy for $B$, implying that $A$ prefers a change of the contract (e.g. by pressure of strike) above all and no certification or threat of sanction.

The preference order ranks the strategy profiles according to the player's preference over the associated outcomes. The order is from left to right, placing the strategy profile that is associated to the most preferred outcome in the left most position. All strategy profiles to the right are strictly less preferred, except if the profiles are connected by a bar, indicating indifference. The preference order for $A$ looks as in table 2 (grey implies that these strategy profiles are considered infeasible). In the first preference order, the outcomes associated with 7 and 3 are equally preferred, and the outcome associated to 12 is least preferred.

Table 2. Preference Vector I

Preference order of $A$
\begin{tabular}{|l||c|c|c|c|c|c|c|c|c|}
\hline Start & $\overline{7} \mid 3$ & 15 & 0 & 1 & 5 & 4 & 13 & 12 \\
\hline End & 3 & 7 & 15 & 0 & 4 & 12 & 1 & 5 & 13 \\
\hline
\end{tabular}

The preference order of $B$ is separated into three different cases. Like for $A$, the first case represents the initial situation of the model. $B$ prefers to offer a non-exploitative contract. Certifying the contract beforehand is slightly preferred to just providing the non-exploitative share to $A$ without contractual certification. The threat of sanctioning $A$ is even less preferred, since $B$ considers this unnecessary if $A$ approves the contract (both groups are aware of their mutual benevolence). The second case is the situation, in which $B$ gained in relative bargaining power. $B$ believes that not fulfilling the contract by $A$ will have no fundamental repercussions, e.g. that $A$ reacts only with a limited violent aggression and the effect of strike is negligible. $B$ also believes that an a priori threat of sanction will push $A$ to abide to the terms of the contract and will limit the violence of $A$ 's reaction. Hence, $B$ prefers above all to exploit $A$ and always to threaten with a sanction to only certifying, which is again preferred to not certifying. $B$ prefers 12 to 15 , since the possible loss from non-fulfilment is expected to be lower than the expected loss from offering a non-exploitative contract. Since $B$ has an interest to maintain his status quo power, strategy profile 0 is least preferred. In the third stage, $B$ is aware that $A$ has no sympathy for $B$. If $A$ does not abide to the contract, the subsequent reaction (i.e. a violent general strike or a social revolution) will question and endanger $B$ 's status quo position, since $A$ is likely to win the conflict. Therefore $B$ prefers all those strategy profiles, in which $A$ fulfils the contract, and desires most those, in which $A$ is still exploited. In the case, where $A$ does not intend to abide to the terms of the contract, no certification is preferred to just certifying, which is again preferred to threaten 
with sanctions. The idea is that $B$ fears that the violence of $A$ 's reaction will depend on how much $B$ abused his bargaining power ${ }^{10}$ Consequently $B$ 's preference order is assumed as illustrated in table 3 .

Table 3. Preference Vector II

\begin{tabular}{|l||c|c|c|c|c|c|c|c|c|}
\hline \hline Preference order of $B$ \\
\hline Start & 7 & 3 & 15 & 5 & 1 & 13 & 12 & 4 & 0 \\
\hline Intermediate & 13 & 5 & 1 & 12 & 15 & 7 & 3 & 4 & 0 \\
\hline End & 13 & 5 & 1 & 15 & 7 & 3 & 0 & 4 & 12 \\
\hline
\end{tabular}

\subsection{Stability Analysis without Mis-perception}

It is required to carry out a static analysis of the game before proceeding to a dynamic representation. Based on the given assumptions, 4 different games can be derived for the static model. The first is the case, in which both parties have preferences given by "start". The second is defined by $A$ 's preference order in "start" and $B$ 's preference order as in "intermediate". This implies a situation, in which $B$ has sufficient bargaining power to exploit, but $A$ has insufficient power to successfully demand a change of the contract. Similarly, the next two stages are then given by preferences "end-intermediate" and "end-end". The solution of every static game is represented for efficiency in the tabular form (for a detailed description of the derivation of this form, refer to Ille, 2012; Fraser and Hipel, 1984). The stabilities are indicated for both player groups with $u$ - unstable, $r$ - rationally, $s$ - sequentially stable. These stabilities are derived according to conditions 3 and 4 and all those profiles, which are stable for both groups, define an equilibrium of the game. The first static game and its analysis are given in table 4 below.

The $D P$ s are derived as described from condition 2. The DPs below a certain strategy profile are vertically ordered according to their position in the preference vector, i.e. the highest $D P$ is most preferred by this player group. Strategy profiles lacking a $D P$ are rationally stable according to condition 3 . A change from 1 to 0 of A, i.e. a switch from strategy Abide to not Abide given B's strategy Exploitation, no Certification, no Sanction, entails a subsequent shift of $B$ to 12 or 4 . Both are less preferred than the outcome associated to the original strategy profile 1 . Condition 4 is fulfilled for 1 and player $A$. A shift of $A$ from 4 to 5 causes a subsequent shift of $B$ to 7,3 or 15 , of which all rank higher in the preference order. Neither condition 3 nor 4 are satisfied for 4 . In the same fashion the remaining stabilities are calculated for $A$ and $B$. In the first static game only strategy profile $7=($ Abide;

\footnotetext{
${ }^{10} \mathrm{~A}$ non-violent change of the contract is preferred to an act of violence that will cause additional costs for $B$.
} 
Table 4. First static game

\begin{tabular}{|l||c|c|c|c|c|c|c|c|c|}
\hline \multicolumn{10}{|c|}{ start-start } \\
\hline \hline overall stability & $\mathrm{E}$ & $\mathrm{x}$ & $\mathrm{x}$ & $\mathrm{x}$ & $\mathrm{x}$ & $\mathrm{x}$ & $\mathrm{x}$ & $\mathrm{x}$ & $\mathrm{x}$ \\
\hline \hline \hline stability for $A$ & $\mathrm{r}$ & $\mathrm{r}$ & $\mathrm{r}$ & $\mathrm{r}$ & $\mathrm{s}$ & $\mathrm{r}$ & $\mathrm{u}$ & $\mathrm{r}$ & $\mathrm{u}$ \\
\hline$A$ 's preference order & $\overline{7}$ & 3 & 15 & 0 & 1 & 5 & 4 & 13 & 12 \\
\hline DPs & & & & & 0 & & 5 & & 13 \\
\hline \hline stability for $B$ & $\mathrm{r}$ & $\mathrm{u}$ & $\mathrm{u}$ & $\mathrm{u}$ & $\mathrm{u}$ & $\mathrm{u}$ & $\mathrm{r}$ & $\mathrm{u}$ & $\mathrm{u}$ \\
\hline$B$ 's preference order & 7 & 3 & 15 & 5 & 1 & 13 & 12 & 4 & 0 \\
\hline DPs & & 7 & 7 & 7 & 7 & 7 & & 12 & 12 \\
& & & 3 & 3 & 3 & 3 & & & 4 \\
& & & 15 & 15 & 15 & & & \\
& & & & 5 & 5 & & & \\
& & & & & 1 & & & \\
\hline
\end{tabular}

no Exploitation,Certification) is stable for both groups. Hence, the contract is defined by a fair cooperation. The solution to the second static game is shown in table 5 .

Table 5. Second static game

\begin{tabular}{|c|c|c|c|c|c|c|c|c|c|}
\hline \multicolumn{10}{|c|}{ start-intermediate } \\
\hline overall stability & $\mathrm{X}$ & $\mathrm{x}$ & $\bar{x}$ & $\mathrm{x}$ & $\bar{x}$ & $\bar{x}$ & $\overline{\mathrm{x}}$ & $\bar{E}$ & $\overline{\mathrm{x}}$ \\
\hline stability for $A$ & $\mathrm{r}$ & $\mathrm{r}$ & $\mathrm{r}$ & $\mathrm{r}$ & $\mathrm{s}$ & $\mathrm{r}$ & s & $\mathrm{r}$ & $\mathrm{u}$ \\
\hline A's preference order & $\overline{7}$ & $\overline{3}$ & 15 & 0 & 1 & 5 & 4 & 13 & 12 \\
\hline DPs & & & & & 0 & & 5 & & 13 \\
\hline stability for $B$ & $\mathrm{r}$ & $\mathrm{u}$ & $\mathrm{u}$ & $\mathrm{r}$ & $\mathrm{u}$ & $\mathrm{u}$ & $\mathrm{u}$ & $\mathrm{u}$ & $\mathrm{u}$ \\
\hline$B$ 's preference order & 13 & 5 & 1 & 12 & 15 & 7 & 3 & 4 & 0 \\
\hline DPs & & 13 & $\begin{array}{c}13 \\
5\end{array}$ & & $\begin{array}{c}13 \\
5 \\
1\end{array}$ & $\begin{array}{c}13 \\
5 \\
1 \\
15\end{array}$ & $\begin{array}{c}13 \\
5 \\
1 \\
15 \\
7\end{array}$ & 12 & $\begin{array}{c}12 \\
4\end{array}$ \\
\hline
\end{tabular}

The stable equilibrium of the second game in table 5 is defined by 13=(Abide; Exploitation, Certification, Sanction). Table 6 describes the third static game, in which $A$ prefers a social change to the status quo situation, but $B$ believes to have sufficient bargaining power to prevent such a change 11 The equilibrium of the third game in table 6 is defined by $12=($ Not Abide; Exploitation, Certification, Sanction $) . A$ asks for a social change, but $B$ is unwilling to give up his status quo position. The last game, in which the probability of a successful social change is high, is depicted in table 7

\footnotetext{
${ }^{11}$ In the game context, this period could be interpreted as an attempt of social turnover.
} 
Table 6. Third static game

\begin{tabular}{|l||c|c|c|c|c|c|c|c|c|}
\hline \multicolumn{10}{|c|}{ end-intermediate } \\
\hline \hline overall stability & & & & $\mathrm{x}$ & $\mathrm{x}$ & $\mathrm{E}$ & $\mathrm{x}$ & $\mathrm{x}$ & $\mathrm{x}$ \\
\hline \hline \hline stability for $A$ & & & & $\mathrm{r}$ & $\mathrm{r}$ & $\mathrm{r}$ & $\mathrm{u}$ & $\mathrm{u}$ & $\mathrm{u}$ \\
\hline$A$ 's preference order & 3 & 7 & 15 & 0 & 4 & 12 & 1 & 5 & 13 \\
\hline DPs & & & & & & & 0 & 4 & 12 \\
\hline \hline stability for $B$ & $\mathrm{r}$ & $\mathrm{s}$ & $\mathrm{s}$ & $\mathrm{r}$ & $\mathrm{u}$ & $\mathrm{u}$ & $\mathrm{u}$ & $\mathrm{u}$ & $\mathrm{u}$ \\
\hline$B$ 's preference order & 13 & 5 & 1 & 12 & 15 & 7 & 3 & 4 & 0 \\
\hline DPs & & 13 & 13 & & 13 & 13 & 13 & 12 & 12 \\
& & & 5 & & 5 & 5 & 5 & & 4 \\
& & & & & 1 & 1 & 1 & & \\
& & & & & & 15 & 15 & & \\
& & & & & & & 7 & & \\
\hline
\end{tabular}

Table 7. Fourth static game

\begin{tabular}{|l||c|c|c|c|c|c|c|c|c|}
\hline \multicolumn{7}{|c|}{ end-end } \\
\hline \hline overall stability & & & $\mathrm{E}$ & $\mathrm{E}$ & $\mathrm{x}$ & $\mathrm{x}$ & $\mathrm{x}$ & $\mathrm{x}$ & $\mathrm{x}$ \\
\hline \hline \hline stability for $A$ & & & & $\mathrm{r}$ & $\mathrm{r}$ & $\mathrm{r}$ & $\mathrm{u}$ & $\mathrm{u}$ & $\mathrm{u}$ \\
\hline$A$ 's preference order & 3 & 7 & 15 & 0 & 4 & 12 & 1 & 5 & 13 \\
\hline DPs & & & & & & & 0 & 4 & 12 \\
\hline \hline stability for $B$ & $\mathrm{r}$ & $\mathrm{s}$ & $\mathrm{s}$ & $\mathrm{s}$ & $\mathrm{u}$ & $\mathrm{u}$ & $\mathrm{r}$ & $\mathrm{u}$ & $\mathrm{u}$ \\
\hline$B$ 's preference order & 13 & 5 & 1 & 15 & 7 & 3 & 0 & 4 & 12 \\
\hline DPs & & 13 & 13 & 13 & 13 & 13 & & 0 & 0 \\
& & & 5 & 5 & 5 & 5 & & & 4 \\
& & & 1 & 1 & 1 & & & \\
& & & & 15 & 15 & & & \\
& & & & & 7 & & & \\
\hline
\end{tabular}

The equilibrium is defined by $0=($ Not Abide; Exploitation $)$ or $15=($ Abide, No Exploitation, Certification, Sanction). The former is the situation, in which $A$ and $B$ do not reach a mutual consent regarding their contract. The second defines a situation similar to the previous game in table 6, but $B$ prefers to offer a non-exploitative contract fearing the eventual repercussion. Based on the original interpretation of the game, the strategy profile symbolised by 0 is regarded as a period, during which the social or work contract is rewritten. Since $A$ deems 15 infeasible, it will not choose action Abide. The static model describes a player population moving through the following states: $(7 \rightarrow 13 \rightarrow 12 \rightarrow 0)$. 


\subsection{Hypergames}

A hypergame occurs, whenever some player $j$ is wrong about the true nature of game $G$ and perceives a game that either or both differs with respect to the actual preference order or to the available strategies in the sets $S_{-j}$ of the other players. Define player $i$ 's strategy set and preference order by the vector $V_{i}=\left\{S_{i}, U_{i}\right\}$. A non-cooperative nplayer game can be represented by $G=\left(V_{1}, V_{2}, . ., V_{n}\right)$. If one or more players misperceive the underlying rules, game $G$ for player $j$ is given by $G_{j}=\left(V_{1 j}, V_{2 j}, . ., V_{n j}\right)$ and hence, a first level hypergame is defined as $H=\left(G_{1}, G_{2}, \ldots, G_{n}\right)$. If other players are aware of the faulty perception of player $j$, the game turns into a second level hypergame, where the game for player $j$ is defined by an individual first level hypergame $H_{q}=\left(G_{1 q}, G_{2 q}, \ldots, G_{n q}\right)$. Consequently the second level hypergame is represented by $H^{2}=\left(H_{1}, H_{2}, \ldots, H_{n}\right)$. The reasoning can be continued for higher level hypergames. A third level hypergame would occur in the case, where some player erroneously perceive another players misperception, which is again recognised by other players. The third level hypergame will be represented by $H^{3}=\left(H_{1}^{2}, H_{2}^{2}, \ldots, H_{n}^{2}\right)$. In the case of two players with $i=A, B$ a first level hypergame is characterised by $H=\left(G_{A}, G_{B}\right)$. A third level hypergame will have the form

$$
H^{3}=\left(H_{A}^{2}, H_{B}^{2}\right)=\left\{\begin{array}{ll}
H_{A A} & H_{B A} \\
H_{A B} & H_{B B}
\end{array}\right\}=\left\{\begin{array}{llll}
\left(G_{A A A}\right. & \left.G_{B A A}\right) & \left(G_{A B A}\right. & G_{B B A}
\end{array}\right) .
$$

The equilibria of a first level hypergame depend on the stability of each player's strategies within their individual games. The set of equilibria is defined by those strategy profiles that are stable according the the individual perception given by the individual stabilities in $H=\left(G_{A}, G_{B}\right)$, i.e. by the strategy profiles stable both in $V_{A A}$ and $V_{B B}$.

Before developing the dynamic representation, the following short subsection will consider a first level hypergame. This will be of interest, when the dynamic representation with state dependent transition probabilities will be developed. In comparison with the results obtained here, the state dependent approach can dynamically model hypergames 12 Suppose that $B$ overestimates the benevolence of $A$ and its general willingness to accept any contract. On the other hand, $A$ wrongly estimates $B$ 's bargaining power and intentions. Both groups believe to be playing entirely different games (see table 8), illustrating the situation, in which $B$ believes to be still playing "start-start", and $A$ to be playing "end-end". The stabilities for the individual strategy profiles in the two games can be directly taken from the tables 4 and 7 , and are stated again in the upper half of table 8 for simplicity. The equilibria of this hypergame are given by the stabilities of each group according to its individual game. This is represented in the lower part of the table. Consequently, in this

\footnotetext{
${ }^{12}$ This refers also to an n-level hypergame, since, as illustrated in Article 4, a hypergame of any level can be represented by a first level hypergame.
} 
case the strategy profile would be either 7 or 12, unlike only 7 or 0 as expected by $B$ or by $A$, respectively. If $A$ assumes 3,7 , and 15 infeasible and will not choose action Abide, the final and only equilibrium of the game is defined by 12 .

Table 8. First Level Hypergame: Mutual Mis-perception of the Game

\begin{tabular}{|c|c|c|c|c|c|c|c|c|c|}
\hline \multicolumn{10}{|c|}{ "end-end" - $A$ 's perception } \\
\hline stability for $A$ & & & & $\overline{\mathrm{r}}$ & $\overline{\mathrm{r}}$ & $\overline{\mathrm{r}}$ & $\overline{\mathrm{u} u}$ & $\overline{\mathrm{u}}$ & $\overline{\mathrm{u}}$ \\
\hline$A$ 's preference order & 3 & 7 & 15 & 0 & 4 & 12 & 1 & 5 & 13 \\
\hline stability for $B$ & $\mathrm{r}$ & $\mathrm{s}$ & $\mathrm{s}$ & $\mathrm{s}$ & $\mathrm{u}$ & $\mathrm{u}$ & $\mathrm{r}$ & $\mathrm{u}$ & $\mathrm{u}$ \\
\hline$B$ 's preference order & 13 & 5 & 1 & 15 & 7 & 3 & 0 & 4 & 12 \\
\hline \multicolumn{10}{|c|}{ "start-start" - $B$ 's perception } \\
\hline stability for $A$ & $\overline{\mathrm{r}}$ & $\overline{\mathrm{r}}$ & $\overline{\mathrm{r}}$ & $\overline{\mathrm{r}}$ & 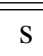 & $\overline{\mathrm{r}}$ & $\overline{\bar{u}}$ & $\overline{\mathrm{r}}$ & $\overline{\mathrm{u}} \mathrm{u}$ \\
\hline$A$ 's preference order & \multicolumn{2}{|c|}{$\overline{\overline{713}}$} & 15 & 0 & 1 & 5 & 4 & 13 & 12 \\
\hline stability for $B$ & $\mathrm{r}$ & $\mathrm{u}$ & $\mathrm{u}$ & $\mathrm{u}$ & $\mathrm{u}$ & $\mathrm{u}$ & $\mathrm{r}$ & $\mathrm{u}$ & $\mathrm{u}$ \\
\hline$B$ 's preference order & 7 & 3 & 15 & 5 & 1 & 13 & 12 & 4 & 0 \\
\hline \multicolumn{10}{|c|}{ Combining $A$ 's and $B$ 's Stability } \\
\hline overall stability & & 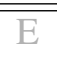 & & $\overline{\mathrm{x}}$ & $\overline{\mathrm{XX}}$ & $\bar{E}$ & $\overline{\mathrm{x}}$ & $\bar{x}$ & $\bar{x}$ \\
\hline stability for $A$ & & & & $\mathrm{r}$ & $\mathrm{r}$ & $\mathrm{r}$ & $\mathrm{u}$ & $\mathrm{u}$ & $\mathrm{u}$ \\
\hline As preference order & 3 & 7 & 15 & 0 & 4 & 12 & 1 & 5 & 13 \\
\hline stability for $B$ & $\mathrm{r}$ & $\mathrm{u}$ & $\mathrm{u}$ & $\mathrm{u}$ & $\mathrm{u}$ & $\mathrm{u}$ & $\mathrm{r}$ & $\mathrm{u}$ & $\mathrm{u}$ \\
\hline$B$ 's preference order & 7 & 3 & 15 & 5 & 1 & 13 & 12 & 4 & 0 \\
\hline
\end{tabular}

\subsection{Dynamic representation}

Though the analysis of the game in its static form already indicates the possible equilibria that might occur during various stages of interaction, a dynamic representation, which can be controlled to continuously move between the various preference orders, is preferable. As a first step, the individual transition matrix for each group must be derived on the basis of the static analysis. The individual transition matrices will be used to form the transition matrix $T$ for the entire game. A definition of the individual transition matrices, solely on the basis of the initial (start-start) and the final (end-end) game, should suffice as an example.

For a games with $f$ strategy profiles, let a state $X_{t}$ be defined by the probability distribution of the strategy profiles in time $t$ with dimension $f \times 1$, i.e. by a vector, where each component indicates the likelihood of a strategy profile at time $t$ over the entire set $S$. The Markov process is determined by

$$
X_{t}=T X_{t-1}
$$


and $T$ is the transition matrix of dimension $f \times f$ that describes the transition probability of moving from strategy profile $x$ in period $t-1$ to $y$ in $t$. Under the condition that the transition matrix $\mathrm{T}$ is time homogeneous the Markov process is defined by $X_{t}=T^{t} X_{0}$. Two variants can be used as a basis to model preferential change:

Variant 1: As a first assumption, consider that players exogenously change their expectations about the game they are playing. Since each individual transition matrix should reflect the preference order in both games, the original approach needs some adaptation. Define a "transition probability" $\alpha$ and $\gamma$. The first refers to the preference order of $A$, the latter to preference order of $B$. Define the transition probability for $A$ in such a way that $1-\alpha$ gives the probability of being in a state defined by game (start-start) and hence, $\alpha$ is the probability of being in state defined by game (end-end). A continuum of states, defined by a specific value of the transition probability, can be thus described, ranging from the stabilities of $A$ as in start, start $(\alpha=0)$ to its stabilities as in end, end $(\alpha=1)$. The transition probabilities hence enable us to shift between the preference orders of each group. The transition matrix $T_{A}$ for group $A$ is given in table 9 .

Table 9. Individual Transition Matrix with identical Perception

\begin{tabular}{|c||c|c|c|c|c|c|c|c|c|}
\hline \multicolumn{1}{|c|}{ Transition Matrix for $A$} \\
\hline \hline & $\mathbf{0}$ & $\mathbf{1}$ & $\mathbf{3}$ & $\mathbf{4}$ & $\mathbf{5}$ & $\mathbf{7}$ & $\mathbf{1 2}$ & $\mathbf{1 3}$ & $\mathbf{1 5}$ \\
\hline \hline $\mathbf{0}$ & 1 & $\alpha$ & 0 & 0 & 0 & 0 & 0 & 0 & 0 \\
\hline $\mathbf{1}$ & 0 & $1-\alpha$ & 0 & 0 & 0 & 0 & 0 & 0 & 0 \\
\hline $\mathbf{3}$ & 0 & 0 & 1 & 0 & 0 & 0 & 0 & 0 & 0 \\
\hline $\mathbf{4}$ & 0 & 0 & 0 & 1 & $\alpha$ & 0 & 0 & 0 & 0 \\
\hline $\mathbf{5}$ & 0 & 0 & 0 & 0 & $1-\alpha$ & 0 & 0 & 0 & 0 \\
\hline $\mathbf{7}$ & 0 & 0 & 0 & 0 & 0 & 1 & 0 & 0 & 0 \\
\hline $\mathbf{1 2}$ & 0 & 0 & 0 & 0 & 0 & 0 & $\alpha$ & $\alpha$ & 0 \\
\hline $\mathbf{1 3}$ & 0 & 0 & 0 & 0 & 0 & 0 & $1-\alpha$ & $1-\alpha$ & 0 \\
\hline $\mathbf{1 5}$ & 0 & 0 & 0 & 0 & 0 & 0 & 0 & 0 & 1 \\
\hline
\end{tabular}

Though derivation of this matrix is intuitive, it is useful to look at the original preference order and the stabilities of tables 4 and 7 , shown again in table 10 . For simplicity, it is

Table 10. Comparison Initial \& Final Stage

\begin{tabular}{|c|c|c|c|c|c|c|c|c|c|}
\hline \multicolumn{10}{|c|}{ Preference order of $A$ at the initial and final stage } \\
\hline stability for $A$ in initial stage & 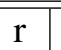 & $\overline{\mathrm{r}}$ & $\mathrm{r}$ & $\overline{\mathrm{r}}$ & 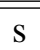 & $\mathrm{r}$ & 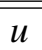 & $\mathrm{r} \mathrm{r}$ & $\overline{\mathrm{u}} \mathrm{u}$ \\
\hline As preference order & $\frac{1}{71}$ & & 15 & 0 & 1 & 5 & 4 & 13 & 12 \\
\hline DPs & & & & & 0 & & 5 & & 13 \\
\hline stability for $A$ in fin & & & & $r$ & $r$ & $r$ & u & u & $\mathrm{u}$ \\
\hline A's preference order & 3 & 7 & 15 & 0 & 4 & 12 & 1 & 5 & 13 \\
\hline DPs & & & & & & & 0 & 4 & 12 \\
\hline
\end{tabular}


generally assumed that a player switches to his most preferred non-sanctioned DP. In both games, strategy profiles $0,3,4,7$, and 15 are stable. Hence, a value of 1 is written along the main diagonal for these strategy profiles. Strategy profile 1 is stable in the initial game, but has a $D P$ and therefore a transition to 0 in the final game. Thus, with probability $1-\alpha, A$ remains in strategy profile 1 , with probability $\alpha$ it switches to strategy profile 0 . The same logic provides the rest of the matrix for the remaining strategy profiles. In the same manner the transition matrix $T_{B}$ in table 11 for $B$ is derived, where $\gamma$ gives the transition probability from the initial preference order to the one in the final state of the game. Hence, $\gamma=0$ describes $B$ 's stabilities in (start, start) and $\gamma=1$ its stabilities in (end, end). Keep in mind that a player can only switch to a non-sanctioned $D P$. The most preferred non-sanctioned $D P$ of $B$ for 3 and 7 is 15 .

Table 11. Individual Transition Matrix with identical Perception

\begin{tabular}{|l||c|c|c|c|c|c|c|c|c|}
\hline \multicolumn{7}{|c|}{ Transition Matrix for $B$} \\
\hline \hline & $\mathbf{0}$ & $\mathbf{1}$ & $\mathbf{3}$ & $\mathbf{4}$ & $\mathbf{5}$ & $\mathbf{7}$ & $\mathbf{1 2}$ & $\mathbf{1 3}$ & $\mathbf{1 5}$ \\
\hline \hline $\mathbf{0}$ & $\gamma$ & 0 & 0 & $\gamma$ & 0 & 0 & $\gamma$ & 0 & 0 \\
\hline $\mathbf{1}$ & 0 & $\gamma$ & 0 & 0 & 0 & 0 & 0 & 0 & 0 \\
\hline $\mathbf{3}$ & 0 & 0 & 0 & 0 & 0 & 0 & 0 & 0 & 0 \\
\hline $\mathbf{4}$ & 0 & 0 & 0 & 0 & 0 & 0 & 0 & 0 & 0 \\
\hline $\mathbf{5}$ & 0 & 0 & 0 & 0 & $\gamma$ & 0 & 0 & 0 & 0 \\
\hline $\mathbf{7}$ & 0 & $1-\gamma$ & $1-\gamma$ & 0 & $1-\gamma$ & $1-\gamma$ & 0 & $1-\gamma$ & $1-\gamma$ \\
\hline $\mathbf{1 2}$ & $1-\boldsymbol{\gamma}$ & 0 & 0 & $1-\gamma$ & 0 & 0 & $1-\gamma$ & 0 & 0 \\
\hline $\mathbf{1 3}$ & 0 & 0 & 0 & 0 & 0 & 0 & 0 & $\gamma$ & 0 \\
\hline $\mathbf{1 5}$ & 0 & 0 & $\gamma$ & 0 & 0 & $\gamma$ & 0 & 0 & $\gamma$ \\
\hline
\end{tabular}

Subsequently, these two matrices are combined into a single transition matrix $T$ (see table 12). The derivation of this matrix is more complicated than the derivation of the individual transition matrices. By looking at the transition matrix for $A$, it can be seen that $A$ stays at

Table 12. Final Transition Matrix

\begin{tabular}{|c|c|c|c|c|c|c|c|c|c|}
\hline \multicolumn{10}{|c|}{ Combined Transition Matrix for the Game } \\
\hline & $\overline{\mathbf{0}}$ & $\overline{1}$ & $\overline{3}$ & 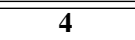 & 5 & 7 & 12 & 13 & 15 \\
\hline$\overline{\mathbf{0}}$ & $\bar{\gamma}$ & $\overline{\gamma \alpha \alpha}$ & 0 & $\gamma \alpha$ & $\overline{0}$ & 0 & $\alpha \alpha \gamma$ & 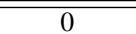 & $\overline{0}$ \\
\hline 1 & 0 & $(1-\alpha) \gamma$ & 0 & $(1-\alpha) \gamma$ & 0 & 0 & $(1-\alpha) \gamma$ & 0 & 0 \\
\hline 3 & 0 & 0 & 0 & 0 & 0 & 0 & 0 & 0 & 0 \\
\hline 4 & 0 & $\alpha(1-\gamma)$ & 0 & 0 & $\alpha$ & 0 & 0 & $\alpha(1-\gamma)$ & 0 \\
\hline 5 & 0 & 0 & 0 & 0 & $(1-\alpha) \gamma$ & 0 & 0 & 0 & 0 \\
\hline 7 & 0 & $(1-\alpha)(1-\gamma)$ & $1-\gamma$ & 0 & $(1-\alpha)(1-\gamma)$ & $1-\gamma$ & 0 & $(1-\alpha)(1-\gamma)$ & $1-\gamma$ \\
\hline 12 & $1-\gamma$ & 0 & 0 & $\alpha(1-\gamma)$ & 0 & 0 & $\alpha(1-\gamma)$ & $\alpha \gamma$ & 0 \\
\hline 13 & 0 & 0 & 0 & $(1-\alpha)(1-\gamma)$ & 0 & 0 & $(1-\alpha)(1-\gamma)$ & $(1-\alpha) \gamma$ & 0 \\
\hline 15 & 0 & 0 & $\gamma$ & 0 & 0 & $\gamma$ & 0 & 0 & $\gamma$ \\
\hline
\end{tabular}

strategy profile 0 with certainty. Therefore group $A$ will choose $(0,-,-,-)^{T}$ with probability $1{ }^{13} B$ stays in 0 with probability $\gamma$ and switches to 12 with probability $1-\gamma$. Accordingly,

\footnotetext{
${ }^{13}$ Remember that the strategy profile can also be written as a vector in binary code. Here $A$ is only able to change the first value of the vector. A slash indicates that the group cannot affect these values.
} 
group $B$ is expected to choose $(-, 0,0,0)^{T}$ or $(-, 0,1,1)^{T}$. The expected strategy profiles for the final transition matrix are given by $(0,0,0,0)^{T}$ with probability $1 \times \gamma$, and $(0,0,1,1)^{T}$ with probability $1 \times(1-\gamma)$. Strategy profile 12 stays with probability $\alpha$ in 12 and with probability $(1-\alpha)$ switches to 13 . Hence, $A$ chooses to accept the contract with probability $(1-\alpha)$ and not to with probability $\alpha$. $B$ changes to strategy profile 0 , i.e. it chooses $(-, 0,0,0)^{T}$, with probability $\gamma$, and stays in 12 , defined by $(-, 0,1,1)^{T}$, with probability $(1-$ $\gamma)$. Hence, strategy profile 12 is followed by 0 with probability $\alpha \times \gamma$, by 1 with probability $(1-\alpha) \times \gamma$, by 12 with probability $\alpha \times(1-\gamma)$, and by 13 with probability $(1-\alpha) \times(1-\gamma)$. The calculation used to find the equilibria in the joint transition matrix is simplified by the following equation:

$$
\bar{q}=\sum_{i=1}^{x} \dot{o}_{i}-(x-1) \dot{q},
$$

where $\bar{q}$ defines the value of the new equilibrium, $x$ the number of player groups (here $x=2$ ), and $\dot{o}_{i}$ the value of the $D P$ of group $i$ from strategy profile $q$ given by value $\dot{q}$.

Some transitions would lead to strictly dominated strategy profiles, which have been ruled out before. Equation 6 is thus inapplicable in these cases. Strategy profile 1, for example, is followed by strategy profile $(0,1,1,0)^{T}$ with probability $\alpha \times(1-\gamma)$. Yet, this strategy profile is considered infeasible. There is, however, a logical solution. The reason for ruling out strategy profiles $(0,1,-,-)^{T}$ in the first place, has been that if $A$ does not abide, a strategy including a non-exploitative contract was assumed strictly dominated. Hence, it is reasonable to suppose that the group will cause the strategy profile to switch to $(0,0,1,0)^{T}$, namely 4 . Therefore the probabilities are added to strategy profile 414

The two games and the transition between them can be represented by an adapted version of equation 5. Each state is determined by a unique transition matrix $T_{\alpha, \beta}$; a variant of the original transition matrix $T$, given the unique values of the transition probabilities $\alpha(t)$ and $\gamma(t)$ in each period $t$. Hence, a state in period $t$ is defined by $X_{t}=T_{\alpha, \beta}^{t} X_{0}$. Notice that the transition probabilities disregard the probability a player assigns to certain strategies in

${ }^{14}$ Though contrary to the initial assumption, it can be argued that $B$
In that case, strategy profile 6 can be substituted by $7 . \quad$ The matrix
\begin{tabular}{|l||c|c|c|c|c|c|c|c|c|}
\hline \begin{tabular}{l}
\hline Combined individual Transition Matrices of each group \\
\hline \hline
\end{tabular} & $\mathbf{0}$ & $\mathbf{1}$ & $\mathbf{3}$ & $\mathbf{4}$ & $\mathbf{5}$ & $\mathbf{7}$ & $\mathbf{1 2}$ & $\mathbf{1 3}$ & $\mathbf{1 5}$ \\
\hline $\mathbf{0}$ & $\gamma$ & $\alpha \gamma$ & 0 & $\gamma \alpha$ & 0 & 0 & $\alpha \gamma$ & 0 & 0 \\
\hline $\mathbf{1}$ & 0 & $(1-\alpha) \gamma$ & 0 & $(1-\alpha) \gamma$ & 0 & 0 & $(1-\alpha) \gamma$ & 0 & 0 \\
\hline $\mathbf{3}$ & 0 & 0 & 0 & 0 & 0 & 0 & 0 & 0 & 0 \\
\hline $\mathbf{4}$ & 0 & 0 & 0 & 0 & $\alpha \gamma$ & 0 & 0 & 0 & 0 \\
\hline $\mathbf{5}$ & 0 & 0 & 0 & 0 & $(1-\alpha) \gamma$ & 0 & 0 & 0 & 0 \\
\hline $\mathbf{7}$ & 0 & $(1-\gamma)$ & $1-\gamma$ & 0 & $(1-\gamma)$ & $1-\gamma$ & 0 & $(1-\gamma)$ & $1-\gamma$ \\
\hline $\mathbf{1 2}$ & $1-\gamma$ & 0 & 0 & $\alpha(1-\gamma)$ & 0 & 0 & $\alpha(1-\gamma)$ & $\alpha \gamma$ & 0 \\
\hline $\mathbf{1 3}$ & 0 & 0 & 0 & $(1-\alpha)(1-\gamma)$ & 0 & 0 & $(1-\alpha)(1-\gamma)$ & $(1-\alpha) \gamma$ & 0 \\
\hline $\mathbf{1 5}$ & 0 & 0 & $\gamma$ & 0 & 0 & $\gamma$ & 0 & 0 & $\gamma$ \\
\hline
\end{tabular}

Simulations results are similar to what is obtained from applying transition matrix 12 
different states. Probabilities are only defined by the composition of $X_{0}$. Equilibrium 15 is therefore observable, though it has been exogenously assigned zero probability in the analysis, presented in table 7. The vector $X_{0}$, indicating the status quo, needs to be defined $a$ priori for the simulation. Strategy profile 0 is a reasonable assumption to describe a situation at the initial stage of interaction. No form of contract has yet been offered. The player population could also be considered at a turning point. Hence $X_{0}=(1,0,0,0,0,0,0,0,0)^{T}$. Notice that the initial vector can also describe a state with mixed strategy profiles, such as $X_{0}^{\prime}=(0,0.5,0.5,0,0,0,0,0,0)^{T}$, implying society starting at strategy profile 1 and 3 with equal probability of 0.5 .

Figure 1. Game with 2 Groups

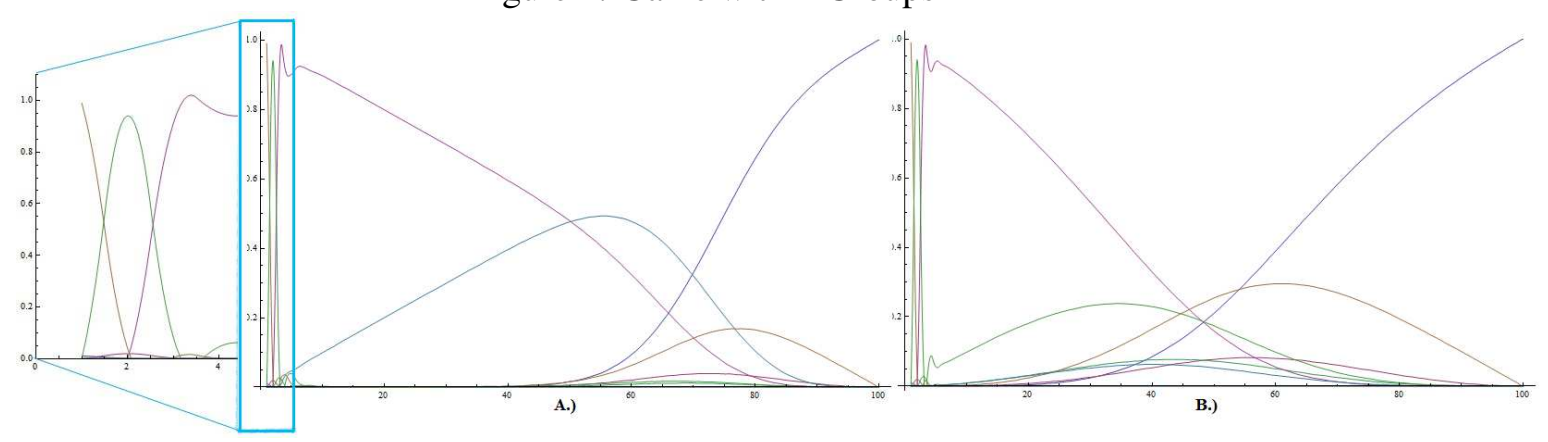

The simulation was conducted for transition probabilities $\alpha(t)=\gamma(t)=\frac{t}{100}$. Figure 1.A.) shows the probability of being in one of the nine possible strategy profiles for $t \in(0,5)$ and $t \in(0,100)$. Table 19 on page 32 provides the rounded probabilities from the first period to the last, in steps of five periods, in order to interpret the figure more easily. Though the two "intermediate games" (i.e. start-intermediate and end-intermediate) were neglected, the dynamic representation already shows a sequence that is alike to the static representation. The player population starts out at 0 ; a point, where initial bargaining begins. It goes immediately to 12 and then to 13 and finally stays at 7 for a longer time. It takes three periods for our player population to reach the first (initially stable) equilibrium. Both groups reach a preliminary agreement on a non-exploitative contract. Strategy profile 15 grows steadily until the middle of the simulation, whereas 7 slowly diminishes. Strategy profile 12 lags behind and obtains a low maximum probability of $11 \%$ in period 80 of the simulation. Finally at the end of the cycle, the society returns to strategy profile 0 ; a social change or reform of the underlying contract $[5$ We thus obtain the sequence $7 \rightarrow 15(\rightarrow 12) \rightarrow 0$. Also notice that the dynamic representation includes first level hypergames. The example

\footnotetext{
${ }^{15}$ Notice that the rise of 15 occurs through the shift of $B$ to its most preferred unsanctioned DP. If it switches from 15 to 13, the dynamics should exactly replicate the results of the static analysis.This assumption contradicts, however, the requirement that a player never chooses a sanctioned DP.
} 
of section 3.2 can be obtained for setting $\alpha=1$ and $\gamma=0$.

Table 13. Reduced Stability Analysis- only most preferred unsanctioned $D P$ is shown

\begin{tabular}{|l||c|c|c|c|c|c|c|c|c|}
\hline \multicolumn{1}{|c|}{ Preference order of $A$ and $B$ as in "start" $P=(1-\alpha)(1-\beta)$} \\
\hline \hline stability for $A$ & $\mathrm{r}$ & $\mathrm{r}$ & $\mathrm{r}$ & $\mathrm{r}$ & $\mathrm{s}$ & $\mathrm{r}$ & $u$ & $\mathrm{r}$ & $\mathrm{u}$ \\
\hline$A$ preference order & $\overline{7 \mid 3}$ & 15 & 0 & 1 & 5 & 4 & 13 & 12 \\
\hline DPs & & & & & & & 5 & & 13 \\
\hline \hline stability for $B$ & $\mathrm{r}$ & $\mathrm{u}$ & $\mathrm{u}$ & $\mathrm{u}$ & $\mathrm{u}$ & $\mathrm{u}$ & $\mathrm{r}$ & $\mathrm{u}$ & $\mathrm{u}$ \\
\hline$B$ 's preference order & 7 & 3 & 15 & 5 & 1 & 13 & 12 & 4 & 0 \\
\hline DPs & & 7 & 7 & 7 & 7 & 7 & & 12 & 12 \\
\hline
\end{tabular}

Preference order of $A$ and $B$ as in "end" $P=\alpha \beta$

\begin{tabular}{|l||c|c|c|c|c|c|c|c|c|}
\hline \hline stability for $A$ & $\mathrm{r}$ & $\mathrm{r}$ & $\mathrm{r}$ & $\mathrm{r}$ & $\mathrm{r}$ & $\mathrm{r}$ & $\mathrm{u}$ & $\mathrm{u}$ & $\mathrm{u}$ \\
\hline$A$ 's preference order & 3 & 7 & 15 & 0 & 4 & 12 & 1 & 5 & 13 \\
\hline DPs & & & & & & & 0 & 4 & 12 \\
\hline \hline stability for $B$ & $\mathrm{r}$ & $\mathrm{s}$ & $\mathrm{s}$ & $\mathrm{s}$ & $\mathrm{u}$ & $\mathrm{u}$ & $\mathrm{r}$ & $\mathrm{u}$ & $\mathrm{u}$ \\
\hline$B$ 's preference order & 13 & 5 & 1 & 15 & 7 & 3 & 0 & 4 & 12 \\
\hline DPs & & & & & 15 & 15 & & 0 & 0 \\
\hline
\end{tabular}

Pr. order of $A$ as in "start" and $B$ as in "end" $P=(1-\alpha) \beta$

\begin{tabular}{|l||c|c|c|c|c|c|c|c|c|}
\hline \hline stability for $A$ & $\mathrm{r}$ & $\mathrm{r}$ & $\mathrm{r}$ & $\mathrm{r}$ & $\mathrm{u}$ & $\mathrm{r}$ & $\mathrm{s}$ & $\mathrm{r}$ & $\mathrm{u}$ \\
\hline$A$ 's preference order & \multicolumn{2}{|c|}{$\overline{7 / 3}$} & 15 & 0 & 1 & 5 & 4 & 13 & 12 \\
\hline DPs & & & & & 0 & & 5 & & 13 \\
\hline \hline stability for $B$ & $\mathrm{r}$ & $\mathrm{u}$ & $\mathrm{u}$ & $\mathrm{u}$ & $\mathrm{u}$ & $\mathrm{u}$ & $\mathrm{r}$ & $\mathrm{u}$ & $\mathrm{u}$ \\
\hline$B$ 's preference order & 13 & 5 & 1 & 15 & 7 & 3 & 0 & 4 & 12 \\
\hline DPs & & 13 & 13 & 13 & 13 & 13 & & 0 & 0 \\
\hline
\end{tabular}

Pr. order of $A$ as in "end" and $B$ as in "start" $P=\alpha(1-\beta)$

\begin{tabular}{|l||c|c|c|c|c|c|c|c|c|}
\hline \hline stability for $A$ & $\mathrm{r}$ & $\mathrm{r}$ & $\mathrm{r}$ & $\mathrm{r}$ & $\mathrm{r}$ & $\mathrm{r}$ & $\mathrm{u}$ & $\mathrm{u}$ & $\mathrm{u}$ \\
\hline$A$ 's preference order & 3 & 7 & 15 & 0 & 4 & 12 & 1 & 5 & 13 \\
\hline DPs & & & & & & & 0 & 4 & 12 \\
\hline \hline stability for $B$ & $\mathrm{r}$ & $\mathrm{u}$ & $\mathrm{u}$ & $\mathrm{u}$ & $\mathrm{u}$ & $\mathrm{u}$ & $\mathrm{r}$ & $\mathrm{u}$ & $\mathrm{u}$ \\
\hline$B$ 's preference order & 7 & 3 & 15 & 5 & 1 & 13 & 12 & 4 & 0 \\
\hline DPs & & 7 & 7 & 7 & 7 & 7 & & 12 & 12 \\
\hline
\end{tabular}


Since a higher level hypergame can be simplified to a first level hypergame as shown above, the dynamic representation is able to incorporate all the properties developed for the static game form. Evidently, in this case 7 and 12 are the only equilibria with value 1 on the main diagonal.

Variant 2: The first variant considered the case, in which players believe that the other players in the game update their preference order in an identical way ${ }^{16}$ Yet previous play, information and expectation can have a different effect on the players' preference orders. As a consequence, players perceive that they shift independently their preference order. For the given example, we thus obtain 4 different games, instead of 2; the same as before, but in addition a game, in which $A$ has preference order "start" and $B$ has preference order "end", and a fourth that represents the inverted case. Table 13 on page 18 illustrates the stabilities of these 4 games. Following the same logic as variant 1, the first game with preference order "start-start" occurs with probability $(1-\alpha)(1-\beta)$, the second defined by "end-end" with probability $\alpha \beta$, the third game defined by "start-end" with probability $(1-\alpha) \beta$, and the fourth defined by "end-start" with probability $\alpha(1-\beta)$.

Based on the transitions in table 13, the individual transition matrix for $\mathrm{A}$ has the shape as in table 14 . The transition probabilities are determined by the DPs in table 13 and the corresponding probability of the game, e.g 0 stays at 0 in all 4 games and thus transition occurs to 0 with probability 1 , strategy profile 1 stays in 1 only in the first game but shifts to 0 in the remaining three, thus transition occurs to 1 with probability $(1-\alpha)(1-\beta)$, and to 0 with probability $1-(1-\alpha)$ $(1-\beta)$. In the same way the transition matrix for $B$ is obtained and represented in table 15

Table 14. Individual Transition Matrix of A: correct perception

\begin{tabular}{|c||c|c|c|c|c|c|c|c|c|}
\hline \multicolumn{2}{|c|}{ Transition Matrix for $A$} \\
\hline \hline & $\mathbf{0}$ & $\mathbf{1}$ & $\mathbf{3}$ & $\mathbf{4}$ & $\mathbf{5}$ & $\mathbf{7}$ & $\mathbf{1 2}$ & $\mathbf{1 3}$ & $\mathbf{1 5}$ \\
\hline \hline $\mathbf{0}$ & 1 & $1-(1-\alpha)(1-\gamma)$ & 0 & 0 & 0 & 0 & 0 & 0 & 0 \\
$\mathbf{1}$ & 0 & $(1-\alpha)(1-\gamma)$ & 0 & 0 & 0 & 0 & 0 & 0 & 0 \\
$\mathbf{3}$ & 0 & 0 & 1 & 0 & 0 & 0 & 0 & 0 & 0 \\
$\mathbf{4}$ & 0 & 0 & 0 & $1-(1-\alpha)(1-\gamma)$ & $\alpha$ & 0 & 0 & 0 & 0 \\
$\mathbf{5}$ & 0 & 0 & 0 & $(1-\alpha)(1-\gamma)$ & $(1-\alpha)$ & 0 & 0 & 0 & 0 \\
$\mathbf{7}$ & 0 & 0 & 0 & 0 & 0 & 1 & 0 & 0 & 0 \\
$\mathbf{1 2}$ & 0 & 0 & 0 & 0 & 0 & 0 & $\alpha$ & $\alpha$ & 0 \\
$\mathbf{1 3}$ & 0 & 0 & 0 & 0 & 0 & 0 & $1-\alpha$ & $1-\alpha$ & 0 \\
$\mathbf{1 5}$ & 0 & 0 & 0 & 0 & 0 & 0 & 0 & 0 & 1 \\
\hline
\end{tabular}

The final transition matrix is obtain in the same way as before, i.e. by combining the two individual transition matrices. Again the transition to strategy profile 6 can occur if equation 6 is applied to calculate the final transitions. As in the earlier variant, the transition probabilities have been attributed to strategy profile 4 (see table 16). A simulation as in variant 1 , with

\footnotetext{
${ }^{16}$ e.g. if $A$ has preferences as in start, it believes that $B$ has also preferences as in start.
} 
$\alpha(t)=\gamma(t)=\frac{t}{100}$, is shown in figure 1 B.) making it directly comparable to the results of variant 1 . Though the initial sequence is identical, as well as the most predominant strategy profiles (i.e. $7=$ violet and $0=$ blue), transition occurs to $13=$ green and $12=$ brown in the intermediate time periods. We obtain a transition that is akin to the static analysis $(7(\rightarrow 13) \rightarrow 12 \rightarrow 0)$.

Both variants suffer, however, from a decisive shortcoming. They model only a "pseudo" dynamic process, since each state is independent of the previous states, and does not add informational value to the static model, though the form of representation is more efficient. Each state $X_{t}$ is defined by $X_{t}=T_{\alpha, \beta}^{t} X_{0}$, i.e. a state in period $t$ is solely determined by the initial state of the world $X_{0}$ and its unique transition matrix $T_{\alpha, \beta}$. Hence, states only differ in the variation of the transition matrix and its exponent. Although, this approach allows to describe a transition between the individual static games and hypergames, and offers the convenient determination of a state without the requirement to calculate previous states, a true dynamic process is defined by $X_{t}=T_{\alpha, \beta} X_{t-1}$. This definition implies that each state defines the "playing ground", i.e. probability distribution of strategy profiles, at the beginning of the next interaction period, based on which players define their best response strategies. Since each state has a unique transition matrix, which shapes the path between states, it obviously holds that $T_{\alpha, \beta} X_{t-1} \neq T_{\alpha, \beta}^{t} X_{0}$, as $T$ is not time homogeneous.

The following analysis is independent of which variant is chosen, since each of the following extensions can be equivalently applied to any variant. Though variant 2 is more apt to model most dynamic interactions, variant 1 offers a less demanding representation, rendering it more accessible to the reader. Consequently, variant 1 is chosen for the subsequent analysis.

Table 15. Individual Transition Matrix of B: correct perception

\begin{tabular}{|c||c|c|c|c|c|c|c|c|c|}
\hline \multicolumn{2}{|c|}{ Transition Matrix for $B$} \\
\hline \hline & $\mathbf{0}$ & $\mathbf{1}$ & $\mathbf{3}$ & $\mathbf{4}$ & $\mathbf{5}$ & $\mathbf{7}$ & $\mathbf{1 2}$ & $\mathbf{1 3}$ & $\mathbf{1 5}$ \\
\hline \hline $\mathbf{0}$ & $\gamma$ & 0 & 0 & $\gamma$ & 0 & 0 & $\gamma$ & 0 & 0 \\
$\mathbf{1}$ & 0 & $\alpha \gamma$ & 0 & 0 & 0 & 0 & 0 & 0 & 0 \\
$\mathbf{3}$ & 0 & 0 & 0 & 0 & 0 & 0 & 0 & 0 & 0 \\
$\mathbf{4}$ & 0 & 0 & 0 & 0 & 0 & 0 & 0 & 0 & 0 \\
$\mathbf{5}$ & 0 & 0 & 0 & 0 & $\alpha \gamma$ & 0 & 0 & 0 & 0 \\
$\mathbf{7}$ & 0 & $1-\gamma$ & $1-\gamma$ & 0 & $1-\gamma$ & $1-\gamma$ & 0 & $1-\gamma$ & $1-\gamma$ \\
$\mathbf{1 2}$ & $1-\gamma$ & 0 & 0 & $1-\gamma$ & 0 & 0 & $1-\gamma$ & 0 & 0 \\
$\mathbf{1 3}$ & 0 & $(1-\alpha) \gamma$ & $(1-\alpha) \gamma$ & 0 & $(1-\alpha) \gamma$ & $(1-\alpha) \gamma$ & 0 & $\gamma$ & $(1-\alpha) \gamma$ \\
$\mathbf{1 5}$ & 0 & 0 & $\alpha \gamma$ & 0 & 0 & $\alpha \gamma$ & 0 & 0 & $\alpha \gamma$ \\
\hline
\end{tabular}


Table 16. Game Transition Matrix: correct perception

\begin{tabular}{|c|c|c|c|c|c|c|c|c|c|}
\hline \multicolumn{10}{|c|}{ Final Transition Matrix } \\
\hline & $\overline{0}$ & $\overline{1}$ & $\overline{3}$ & 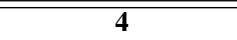 & $\overline{5}$ & 7 & 12 & 13 & 15 \\
\hline$\overline{\overline{0}}$ & $\bar{\gamma}$ & 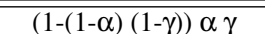 & $\overline{0}$ & 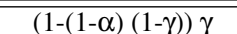 & $\overline{0}$ & $\overline{0}$ & $\alpha \alpha \gamma$ & $\overline{0}$ & $\overline{0}$ \\
\hline 1 & 0 & $(1-\alpha)(1-\gamma)(\alpha \gamma)$ & 0 & $(1-\alpha)(1-\gamma) \gamma$ & 0 & 0 & $(1-\alpha) \gamma$ & 0 & 0 \\
\hline 3 & 0 & 0 & 0 & 0 & 0 & 0 & 0 & 0 & 0 \\
\hline 4 & 0 & $(1-(1-\alpha)(1-\gamma))(1-\gamma)$ & 0 & 0 & $\alpha(\alpha \gamma+(1-\gamma))$ & 0 & 0 & $\alpha(1-\gamma)$ & 0 \\
\hline 5 & 0 & 0 & 0 & 0 & $(1-\alpha) \alpha \gamma$ & 0 & 0 & 0 & 0 \\
\hline 7 & 0 & $(1-\alpha)(1-\gamma)(1-\gamma)$ & $1-\gamma$ & 0 & $(1-\alpha)(1-\gamma)$ & $1-\gamma$ & 0 & $(1-\alpha)(1-\gamma)$ & $1-\gamma$ \\
\hline 12 & $1-\gamma$ & $(1-(1-\alpha)(1-\gamma))(1-\alpha) \gamma$ & 0 & $(1-(1-\alpha)(1-\gamma))(1-\gamma)$ & $\alpha(1-\alpha) \gamma$ & 0 & $\alpha(1-\gamma)$ & $\alpha \gamma$ & 0 \\
\hline 13 & 0 & $(1-\alpha)(1-\gamma)(1-\alpha) \gamma$ & $(1-\alpha) \gamma$ & $(1-\alpha)(1-\gamma)(1-\gamma)$ & $(1-\alpha)(1-\alpha) \gamma$ & $(1-\alpha) \gamma$ & $(1-\alpha)(1-\gamma)$ & $(1-\alpha) \gamma$ & $(1-\alpha) \gamma$ \\
\hline 15 & 0 & 0 & $\alpha \gamma$ & 0 & 0 & $\alpha \gamma$ & 0 & 0 & $\alpha \gamma$ \\
\hline
\end{tabular}

\section{Interaction between three parties with endogenous prefer- ences}

This section will take account of the issue raised in the previous section, but will also add some complexity to the underlying group structure. The Conflict Analysis approach has modelled the interaction of two distinct groups. Since the approach is capable of modelling a larger number of player groups, the following subsection will analyse a non-homogeneous group, i.e. it is assumed that the group $A$ consists of two sub-groups. One sub-group is still defined as $A$, the second sub-group is named $C$. In game $\Gamma=\left(S_{A t}, S_{B t}, S_{C t} ; U_{A t}, U_{B t}, U_{C t}\right)$, both sub-groups, $C$ and $A$, have the same relation towards $B$ and decide, whether or not to abide to the rules of the contract.

\subsection{Non-homogeneous group members}

Since there are now three interacting groups, the strategy profile set increases correspondingly with the added strategy set. Following the same logic in ruling out strictly dominated strategies, i.e. strategy profiles $\left((0,-, 1,-,-)^{T},(-, 0,1,-,-)^{T}\right.$, and $\left.(-,-,-, 0,1)^{T}\right)$ with - indicating any value 0 or 1 , there are 15 possible strategy profiles presented in table 17. The same reasoning for the preference order like in the previous section apply. The previous decimal code for the strategy profiles is rewritten as:

\begin{tabular}{|c|c|c|c|c|c|c|c|c|c|}
\hline original value $\rightarrow$ new value & $0 \rightarrow 0,2$ & $1 \rightarrow 1,3$ & $3 \rightarrow 7$ & $4 \rightarrow 8,10$ & $5 \rightarrow 9,11$ & $7 \rightarrow 15$ & $12 \rightarrow 24,26$ & $13 \rightarrow 25,27$ & $15 \rightarrow 31$ \\
\hline
\end{tabular}

Furthermore $A$ and $C$ are assumed, for simplicity, to have the same preference order (since they have the same relation to $B$ ); the corresponding strategy profiles are only mirrored according to the affected group. Hence, for example strategy profile 1 and 2 exchange places in the preference order of $C$ with respect to $A$. Some additional assumptions are necessary for the asymmetric strategy profiles, in which $A$ and $C$ choose a different strategy. 
Table 17. Strategy profile Set

\begin{tabular}{|c|c|c|c|c|c|c|c|c|c|c|c|c|c|c|c|}
\hline \multicolumn{16}{|l|}{$A$ 's options: } \\
\hline Abide & 0 & 1 & 0 & 1 & 1 & 0 & 1 & 0 & 1 & 1 & 0 & 1 & 0 & 1 & 1 \\
\hline \multicolumn{16}{|l|}{ C's options: } \\
\hline Abide & 0 & 0 & 1 & 1 & 1 & 0 & 0 & 1 & 1 & 1 & 0 & 0 & 1 & 1 & 1 \\
\hline \multicolumn{16}{|l|}{$B$ 's options: } \\
\hline No Exploitation & 0 & 0 & 0 & 0 & 1 & 0 & 0 & 0 & 0 & 1 & 0 & 0 & 0 & 0 & 1 \\
\hline Certification & 0 & 0 & 0 & 0 & 0 & 1 & 1 & 1 & 1 & 1 & 1 & 1 & 1 & 1 & 1 \\
\hline Sanction & 0 & 0 & 0 & 0 & 0 & 0 & 0 & 0 & 0 & 0 & 1 & 1 & 1 & 1 & 1 \\
\hline Decimal code & $\overline{\overline{\mathbf{0}}}$ & $\overline{11}$ & 2 & $\overline{3}$ & $\overline{77}$ & 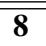 & $\overline{99}$ & 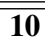 & $\overline{11}$ & $\overline{15}$ & 24 & 25 & $\overline{26}$ & 27 & $\overline{31}$ \\
\hline
\end{tabular}

Generally assume that group $A$ and $C$ prefer to take identical decisions, and for the first preference order assume that both prefer to sign, but not being the only group exploited. For the second preference order, suppose that both favour refusing an exploitative contract at all costs. Hence, the preference order for the three groups can be written as in table 18 (since the focus lies on the dynamic representation, only games "start-start-start" and “end-end-end" are of interest, thus $B$ 's “intermediate" preference order is neglected).

Table 18. Preference Vectors

\begin{tabular}{|c|c|c|c|c|c|c|c|c|c|c|c|c|c|c|c|}
\hline \multicolumn{16}{|c|}{ Preference order of $A$} \\
\hline Start & 7 & 15 & 31 & 0 & 2 & 3 & 1 & 11 & 8 & 9 & 10 & 27 & 25 & 24 & 26 \\
\hline End & 7 & 15 & 31 & 0 & 8 & 24 & 2 & 10 & 26 & 1 & 9 & 25 & 3 & 11 & 27 \\
\hline \multicolumn{16}{|c|}{ Preference order of $C$} \\
\hline Start & 7 & 15 & 31 & 0 & 1 & 3 & 2 & 11 & 8 & 10 & 9 & 27 & 26 & 24 & 25 \\
\hline End & 7 & 15 & 31 & 0 & 8 & 24 & 1 & 9 & 25 & 2 & 10 & 26 & 3 & 11 & 27 \\
\hline \multicolumn{16}{|c|}{ Preference order of $B$} \\
\hline Start & 15 & 7 & 31 & 11 & 3 & 27 & & $\overline{10}$ & & & & & 24 & 8 & 0 \\
\hline End & 27 & 11 & 3 & 31 & 15 & 7 & & $\overline{\overline{10}}$ & & & & & 0 & 8 & 24 \\
\hline
\end{tabular}

The static solution, both of the initial game with the related preference order "start-start-start" and the final game with preference order "end-end-end" is presented in the table 20 on page 33 . The set of equilibria is defined by $E=(1,2,15)$ for the former game, and by $E=(0,31)$ for the latter game. All equilibria are reasonable, but not equally likely. By using the same procedure as before and based on the obtained stabilities, the individual transition matrices can be derived as presented in table 21 on page 34 . Combining the three individual transition matrices results in the final transition matrix $T$ in table 22 on page 35 .

The structure of this matrix provides interesting information about the dynamics of the underlying game. None of the strategy profiles is an absorbing state for all values of $\alpha, \beta$, 
and $\gamma$. Notice that the number of potential equilibria exceeds the five equilibria that have already been determined in the static analysis. The original five equilibria are, however, defined by the transition probability of a single group ${ }^{17}$ The additional potential equilibria can only be stable if two or more groups jointly show the necessary transition probability. If it is assumed that group $A$ and $C$ have similar interests and, hence, approximately the same values for their transition probabilities, the additional equilibria are defined by $3,11,24,27$.

Running the model under the same conditions as before, yields the expected results. Yet, owing to the issue that has been raised in the previous subsection the simulation will take account of the path dependency of each state, i.e. $X_{t}=T_{\omega} X_{t-1}$ (where $T_{\omega}$ is the short notation for $\left.T_{\alpha, \beta, \gamma}\right)$. Since this requires the calculation of each state, I programmed a loop in Mathematica that iterates the calculation process in each period. In addition, I assumed that transition probabilities do not change at the same rate for all groups. For the example, the transition probabilities were defined as $\alpha(t)=0.5 \sin \left[\frac{t-50}{10 \pi}\right]+0.5, \beta(t)=0.5 \sin \left[\frac{t-75}{15 \pi}\right]+0.5$ and $\gamma(t)=0.5 \sin \left[\frac{t-100}{20 \pi}\right]+0.5{ }^{18}$ The simulation was run for 800 periods. The distribution of the strategy profiles from $t=0$ to $t=800$ are presented in figure 2 A.) (values are given in table 23 on page 36 and transition probabilities are illustrated below in 2 B.) ${ }^{19}$

Figure 2. Game with 3 Groups:A.) shows the distribution for each state in $t \in(0,800)$; B.) shows the corresponding values for $\alpha(t)$ (blue), $\beta(t)$ (red), and $\gamma(t)$ (green).

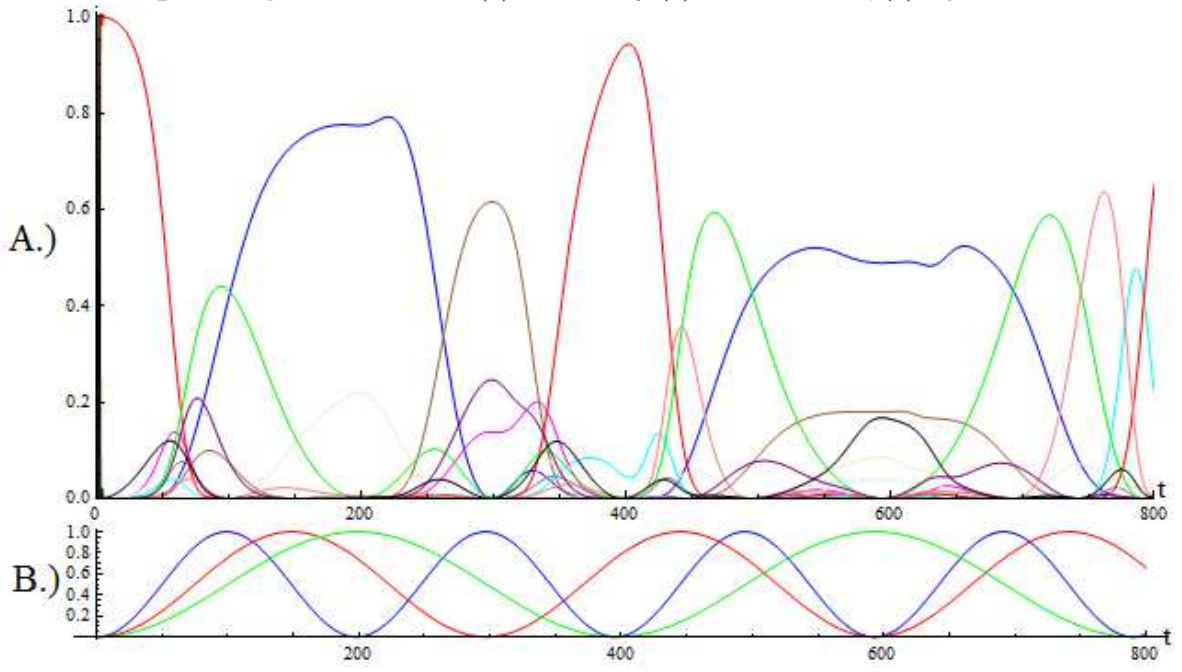

\footnotetext{
${ }^{17}$ Group $A$ determines the stability of 1 , group $C$ the stability of 2 , and group $B$ the stability of 0,15 and 31 .

${ }^{18}$ I have not chosen a nested simulation with linearly increasing and decreasing transition probabilities, since Mathematica showed problems with nested function. A simulation of the form $\alpha(t): \alpha(t)=\frac{t}{n}$ for $t \in(0,100) \cup$ $(200,300) \cup(400,500)$ and $\alpha(t)=1-\frac{t}{n}$ for $t \in(100,200) \cup(300,400) \cup(500,600)$, where $n$ is the upper bound of the corresponding interval was therefore impossible.

${ }^{19}$ This and all following figures use the same colour code: 0- Blue,1- LightBrown, 2-Brown, 3- LightOrange, 7-Yellow, 8-Gray, 9-Cyan, 10-Magenta, 11-LightCyan, 15-Red, 24-Green, 25-Pink, 26-Purple, 27-Black, 31Orange
} 
The non-homogeneous model shows periods of cooperation, exploitation and conflict; represented by the simplified sequence of predominant profiles: 15 (red $) \rightarrow 24$ (green) $\rightarrow$ $0($ blue $) \rightarrow 2($ brown $) \rightarrow 15($ red $) \rightarrow 24($ green $) \rightarrow 0($ blue $) \rightarrow 24($ green $) \rightarrow 25($ pink $) \rightarrow$ $9($ cyan $) \rightarrow 15($ red $)$. This sequence shows that the group, which has a longer cycle (i.e. group $C$ ), accepts the offered contract more quickly and is more prone to exploitation in the initial periods. Yet, the validity of these results is impaired by the exogenous and entirely arbitrary definition of the transition probabilities, which do not exhibit any underlying dependencies. A model that allows for more significant results, requires to endogenise the transition variable. Hence assume that individuals update their preference order based on their experience, when interacting with other players. The strategies, which have been played in previous encounters, will directly affect other-regarding preferences and the bargaining power in future interactions.

\subsection{State Dependent Transition Probabilities}

This section will thus address the issue of endogenising the state dependent individual preference order. Until this point the transition probabilities have not been affected by the outcomes of previous play, but have been defined by an arbitrary relation. Yet, past play will actively influence a player's affections towards another player and the success and the gain from previous play will also determine future bargaining power. An approach that takes account of the effect of past play on the current preference order of a player, is to define a direct relation between the frequency, with which strategy profiles occur, and the transition probability values ${ }^{20}$ For the given case of two different preference orders for an individual group, a higher value of the transition probability illustrates that this group tends towards the second preference order. Lower transition probabilities refer to the first preference order. Thus, the value of each individual transition probability can be assumed to increase in the case, where strategy profiles, which support the conditions underlying the second preference vector of this group/player, occur with higher probability and frequency. On the contrary, transition probability decreases if strategy profiles, which are likely to "shift" individual preferences towards the first preference order, are played with higher probability in the current play and appear more often.

\footnotetext{
${ }^{20}$ Notice, however, that there lies an issue here. Strategy profiles determine outcomes based on the current state, i.e. if the transition probability is high, the outcome that a player associates to a strategy profile is different from the one, he associates in the case of a low transition probability. Hence, on the one hand, the effect of a strategy profile on the transition probability will be determined by the current state. On the other hand, the relative frequency of the strategy profile defines the state. We therefore obtain a circular relation. I will neglect this issue here, since I believe it to be only of minor importance. The strategy profiles that either raise or lower the probability are simply expected to do so under every state. A non-exploitative contract will generally lead to $A$ 's and $C$ 's preferences as in "start-start-start". Similarly an exploitative contract with a threat of sanction will generally have the opposite effect.
} 
Assume a finite number of $f$ different strategy profiles, so that for $\tilde{s}_{k} \in S$, with $k=1, \ldots, f$, a state $X_{t}$ is defined by a vector $X_{t}=\left(x_{1}(t), x_{2}(t), \ldots, x_{f}(t)\right)$, with $\sum_{i=1}^{f} x_{i}(t)=1$, where each $x_{k}(t)$ defines the probability, with which strategy profile $\tilde{s}_{k}$ occurs in $t$. A transition probability $\varphi(t)$, with $\varphi(t)=\alpha(t), \beta(t), \gamma(t)$, can be represented as a function of $X_{t}$. Following the previous line of argument, define a set of strategy profiles $O_{\varphi}^{+}$, which consists of all the strategy profiles that increase the transition probability $\varphi$, since these are expected to lead to preferences as described in the later preference order. Define further another set $O_{\varphi}^{-}$, which consists of all the strategy profiles that diminish the value of $\varphi$, as they induce an individual preference according to the first preference order, implying $O_{\varphi}^{+} \cap O_{\varphi}^{-}=\emptyset$ and $O_{\varphi}^{+} \cup O_{\varphi}^{-} \subseteq S$. Hence, each individual transition probability can be represented as:

$$
\begin{gathered}
\varphi(t)=\varphi(t-1)+\varepsilon_{\varphi}^{+} \sum_{j} x_{j}(t-1)-\varepsilon_{\varphi}^{-} \sum_{h} x_{h}(t-1), \text { where } \\
\varphi(t) \text { is bound to } \varphi(t) \in(0,1) \text {, and } \tilde{s}_{j} \in O_{\varphi}^{+} \text {and } \tilde{s}_{h} \in O_{\varphi}^{-}
\end{gathered}
$$

so that $\sum x_{j}(t-1)+\sum x_{h}(t-1) \leq 1 . \varepsilon_{\varphi}^{+}$and $\varepsilon_{\varphi}^{-}$can be constant or stochastic variables that define the impact of the relative occurrences of a strategy profiles on the transitional change. The game with three groups will again serve as an example.

The assumptions on how to define the sets $O_{\varphi}^{+}$and $O_{\varphi}^{-}$for each transition probability are manifold and so are the resulting dynamics. The following results are only meant as an illustrative example for the approach. The definition of $O_{\varphi}^{+}$and $O_{\varphi}^{-}$are, however, chosen in a plausible way with respect to the context of the game. Remember that the transition probability of $A$ is given by $\alpha(t)$, those of $C$ by $\beta(t)$ and those of $B$ by $\gamma(t)$. For simplicity assume as before that $A$ and $C$ have symmetric preferences. Consequently, sets $O_{\alpha}^{+}$and $O_{\beta}^{+}$, as well as sets $O_{\alpha}^{-}$and $O_{\beta}^{-}$will have a similar structure. They will only differ in those strategy profiles, in which the strategic choices of both groups are different. In such cases the strategy profiles are defined by the mirror image of the corresponding profile for the other group (e.g. the strategy profile denoted by $9=(1,0,0,1,0)^{T}$ in $O_{\alpha}^{+}$corresponds to the strategy profile denoted by $10=(0,1,0,1,0)^{T}$ in $\left.O_{\beta}^{+}\right)$.

Assume that the sympathy for $B$ is reduced in the case, where exploitative and certified contracts occur with higher probability. In the case, where the exploitative contract is not certified, $A$ and $C$ are not legally required to stick to their initial commitment, and these contracts will thus have no effect on the transition probability. An increase in sympathy for $B$ arises only for non-exploitative contracts, where both groups accept or refuse 21 , and where $B$ does not threaten with a sanction in the case of rejection.

The bargaining power of group $B$ is weakened in all those cases, in which both $A$ and

\footnotetext{
${ }^{21}$ Collective rejection is considered as the requirement for re-negotiating the current contract.
} 
$C$ refuse the contract, or in which one group refuses, though $B$ has threatened to sanction the group that does not accept. In these cases, $B$ realises that a sanction is no effective intimidation, thus re-evaluating its bargaining power. Congruently, the bargaining power of $B$ increases in those situation, in which $A$ and $C$ accept the offered contract under any terms. Following this reasoning, the sets are defined as follows: $O_{\alpha}^{+}=$ $(9,11,25,27), O_{\alpha}^{-}=(0,7,8,15)=O_{\beta}^{-}, O_{\beta}^{+}=(10,11,26,27), O_{\gamma}^{+}=(3,7,11,15,27,31)$, and $O_{\gamma}^{-}=(0,8,24,25,26)$. Assume that $\varepsilon_{\varphi}^{-}$and $\varepsilon_{\varphi}^{+}$are equal to 0.2 , which implies that a complete transition from one preference order to the other requires at least 5 interaction periods. Again letting Mathematica simulate the game for 200 periods, shows the result as given in figure 3

Figure 3. Dynamic Representation of Game with 3 Groups - constant $\varepsilon^{-}$and $\varepsilon^{+}$

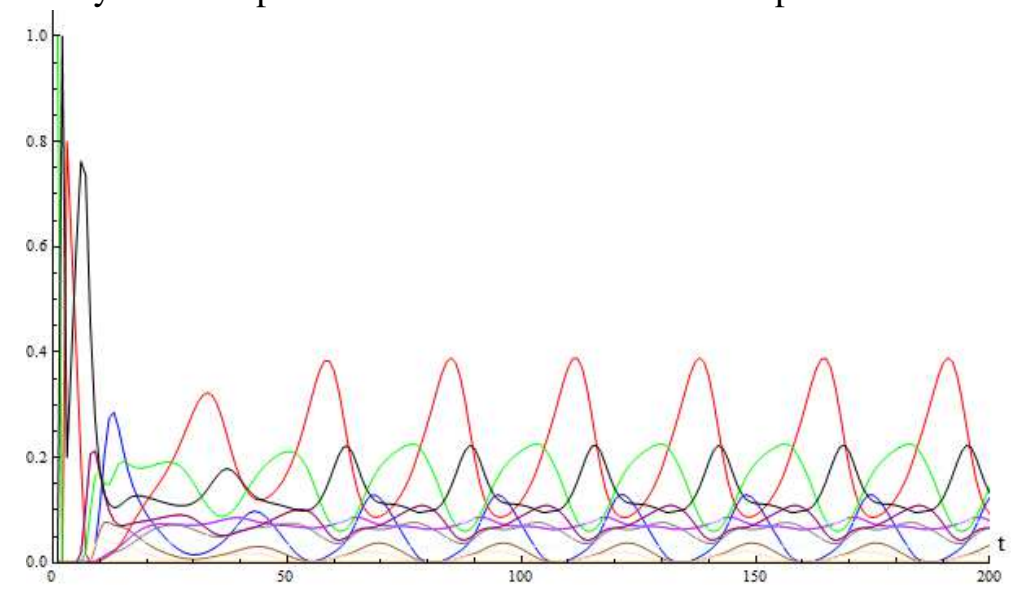

The game is cyclic after about 10 periods. Non-exploitative contracts $15=(1,1,1,1,0)^{T}$ (red) occur at approximately $10-40 \%$, exploitative contracts with sanctions is accepted, $27=(1,1,0,1,1)^{T}$ (black), with approximately $5-25 \%$ and refused, $24=$ $(0,0,0,1,1)^{T}$ (green), with approximately $10-20 \%$ and no contracting, $0=(0,0,0,0,0)^{T}$ (blue), appears with approximately $0-12 \%$.

The regularity, with which these strategy profiles occur does not represent a realistic image of what we would expect the game to look like. The issue here is the constant impact that strategy profiles / outcomes, have on the transition probability, as well as the strict symmetry between group $A$ and $C$. All three groups represent a larger number of players. Since some players will react more violently to certain outcomes than others, idiosyncratic reactions will add noise to the effect of outcomes on the preference order. A similar argument holds for the assumption of strict symmetry between both groups. The following simulation, however, only concentrates on the first aspect. Both $\varepsilon_{\varphi}^{-}$and $\varepsilon_{\varphi}^{+}$are assumed to be continuous and uniformly distributed over 0 to 0.2 for all groups, thus having 
half the same expected value as in the previous simulation. With the addition of added noise in $\varepsilon_{\varphi}^{-}$and $\varepsilon_{\varphi}^{+}$, the simulation has been conducted in the same way as before. The result in figure 4 shows a fairly different and more intuitive result, but the general dynamics are maintained.

Figure 4. Dynamic Representation of Game with 3 Groups $-\varepsilon_{\varphi}^{-}, \varepsilon_{\varphi}^{+} \in(0,0.2)$ : A.) Simulation for 3000 periods, showing only predominant strategy profiles, B.) three dimensional representation- strategy profiles are ordered according to their relative decimal code (from 1 to 15$),$ C.) detailed view of period 2400-2700 with all strategy profiles

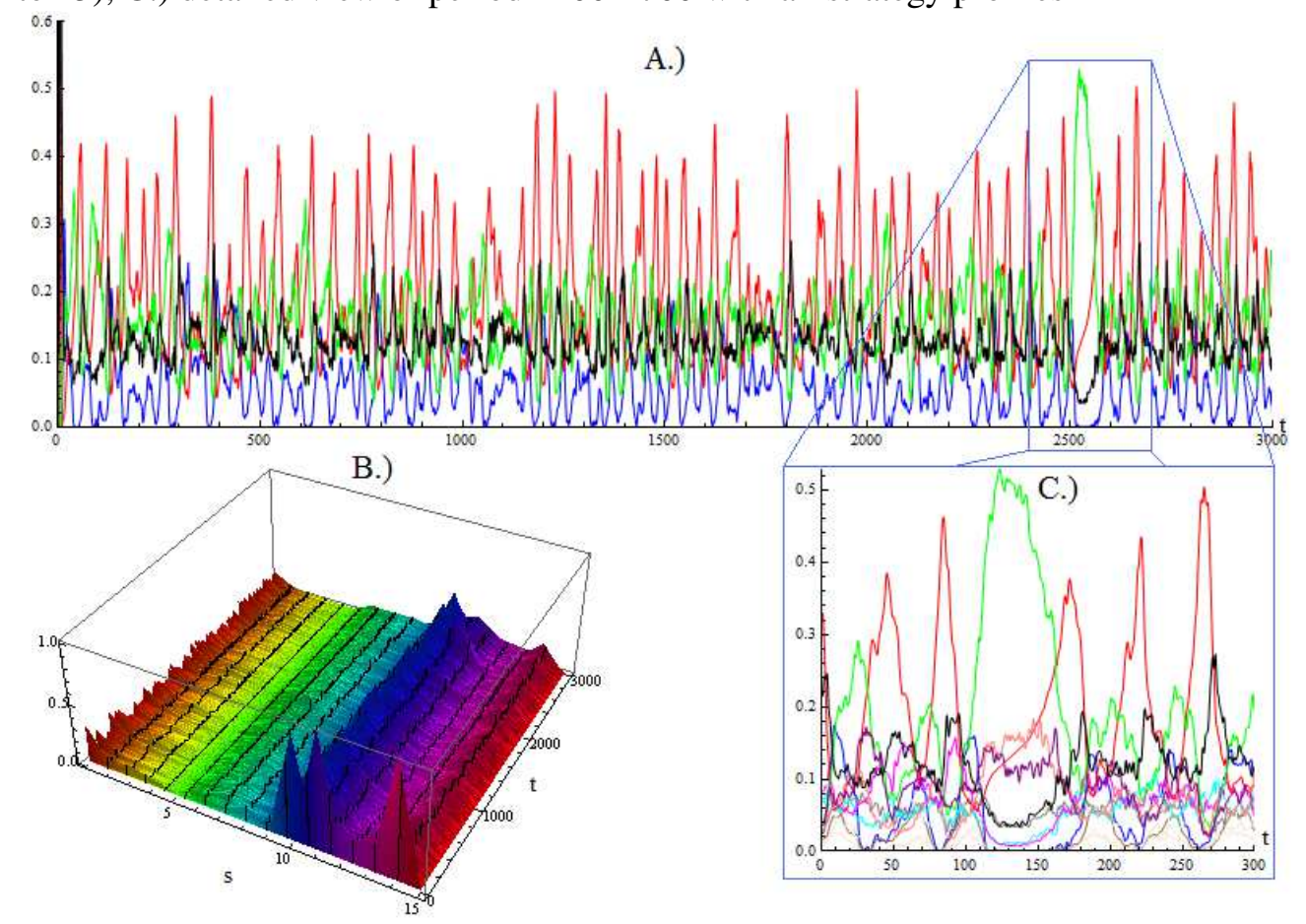

Figure A.) illustrates the simulation for 3000 periods. Since most strategy profiles only occur with very low frequency (on the average below 5\%), I have reduced the presentation to the predominant strategy profiles in figure 3, namely 0 (blue), 15 (red), 24 (green), and 27 (black). Non-exploitative contracts still occur with highest probability. In the stochastic case the exploitative contract with sanction is on average more often rejected (represented by the green line) than accepted (represented by the black line). The relative frequencies of all strategy profiles is better visualised in figure B.). The axis labelled $s$ shows the strategy profiles, as they are simply ranked according to their position in the transition matrix $T 22$ Notice that the colours do not correspond with figure A.). The "combs" at position 1, $10,11,14$, refer to strategy profile $0,15,24$ and 27 , respectively. The highest elevation is at

\footnotetext{
${ }^{22}$ Position/Strategy Profile: 1/0, 2/1, 3/2, 4/3, 5/7, 6/8, 7/9, 8/10, 9/11, 10/15, 11/24, 12/25, 13/26, 14/27, $15 / 31$
} 
the blue ridge (15).

The simulation generated a sequence of periods, in which the exploitative contract has been frequently refused by both $A$ and $C$, though $B$ has threatened with a sanction (see Figure C.) ). Although it seems that a direct correlation between 15 (black) and 24 (green) exists, a look at the transition matrix 22 on page 35 shows that this is not the case. Notice that 25 (pink) and 26(purple) occur more frequent. Looking at the transition matrix 22 on page 35 shows that there are two large blocks of highly connected strategy profiles. The first block is defined by $9,10,11,15$, the second by $24,25,26$. The second block is directly connected to the first by 27 and the first to the second by 8 . Consequently during this period, the game switched from the first to the second block of highly correlated strategy profiles. This switch has occurred through a rise in all transition probabilities leading the system to 8 and 0 . Both strategy profiles are an elements of $O_{\gamma}^{-}$. Hence, the subsequent slump in $\gamma$ pushed the system towards 24. Due to high values of $\alpha$ and $\beta$ and zero value of $\gamma$ the system kept high probability values for this strategy profile, thus creating the short period of "social discontentment".

The values for $\varepsilon_{\varphi}^{+}$and $\varepsilon_{\varphi}^{-}$, and the composition of the sets $O_{\varphi}^{+}$and $O_{\varphi}^{-}$leave much room for further analysis. Different values of $\varepsilon_{\varphi}^{+}$and $\varepsilon_{\varphi}^{-}$for the transition values change the dynamics ${ }^{23}$ Different strategy profiles can be expected to have a different degree of impact. This can be done by splitting $O_{\varphi}^{+}$and $O_{\varphi}^{-}$into various disjoint subsets, and by assigning to each subset a different value of $\varepsilon_{\varphi}^{+}$and $\varepsilon_{\varphi}^{-}$. In addition, the robustness of the results can be tested for changes in the composition of $O_{\varphi}^{+/-}$. The strategy profiles, played in each period, can also be the outcome of a sequence of negotiations. This can be simply included by defining $X_{t}=\left(T_{\alpha, \beta}\right)^{r} X_{t-1}$, where the exponent $r$ simply defines the number of plays that determine the strategy outcome in a given period, i.e. the number of interactions until a conclusion is reached ${ }^{24}$ In short, there are many directions, in which the approach presented in this article can be adapted to various purposes and requirements. Since these changes constitute only a variation of equation 7 , they will not be discussed in the scope of this article.

\footnotetext{
${ }^{23}$ It can be observed, for example, that a group, which exhibits a relatively low impact of the strategy profiles on the transition probabilities, is more prone to exploitation than the other group.

${ }^{24}$ Think of $r$ as the time span of a conference or congress, that determines a treaty or contract for the future period, such as a climate summit or plant bargaining.
} 


\section{Possible Extension and Conclusion}

\subsection{Ideas for an Agent-based model}

As Potts (2000) illustrated, it is more reasonable to model on the basis of what he called "a non-integral framework". We should take account of the incomplete links between agents, incomplete knowledge and necessity to explore technologies and thus the endogeneity of preferences, as equivalent internal constructs to the underlying technologies.

Technologies is synonymous with strategy sets in the context of game theory. An extension of the presented approach should thus include the following: Strategy sets as well as preferences should be able to evolve over time as players interact. Furthermore, the form of inter-group and intra-group links between players should have significant impact on the dynamics of the entire system. Yet, if the effect of connections on the external level (between players and between groups on various levels of aggregation) and on the internal level (in form of changing preferences and strategy options) is accounted for, the model will be unsolvable in closed form. In addition, heterogeneous and boundedly rational agents make it inevitable to use non-arithmetic modelling techniques and complex systems. Weakening the assumption in the described way thus will make it necessary to model by means of agent-based simulations.

In this context the model has been extended in the following way (working paper CEU -Complex Systems- 2009): The model acts on a macro basis (network structure of interactions between agents, groups and classes) and on a micro basis that evolves internally to the individual agent (preferences and resources). Each agent is initially endowed with a constrained set of actions, which evolves over time. They are primary unaware of the results caused by a specific action and also their possible combination into more sophisticated strategies. Thus, new strategies are a function both of previous combinations of actions on the basis of a currently existing action set, and of previous interactions and experiences with other players. The strategy set evolves according to endogenous search heuristics, which have the form of genetically evolving operators (Cross-Over, specification, point mutation - see for example Dosi et al., 1999, 2003; Fagiolo and Dosi, 2003).

Interactions takes place in pairs, matching is random. Strategies and counter-strategies are played until an equilibrium is reached or cyclic behaviour occurs, providing only the fall-back position to each player. With each interaction, a player is increasingly able to associate his and his counterpart's strategies with an outcome, and to form expectations about the sequence of strategies that will be played. Hence, he becomes increasingly aware of his and the other agents' strategy sets (i.e. resources). Furthermore, preferences are incomplete, but adaptive. The pay-off, an individual receives conditional on his chosen strategy, affects his future preference order. Consequently, the evolution of his preference 
vector depends on past experiences and chosen strategies. Since the same holds for his counterpart, an individual cannot guess his partners preferences during early interaction periods. Hence all players are initially unaware of the rules of the game, but understand them better with each interaction. Furthermore, they are also able to learn new strategies and associate outcomes by observing the interactions of their neighbours. A new mutant action and its combination with other actions into a strategy can then spread locally to the neighbours of the individual, who will use this strategy, if successful.

\subsection{Conclusion}

Since the rationality underlying the Conflict Analysis approach is grounded on a sequential reasoning of players, the approach is especially powerful if applied to games that are repeated for an undefined time period, which is obviously the most predominant game type in real-world interactions. Yet, the original approach is very limited in its ability to model and to illustrate the dynamics of such games.

This article targeted the issue by illustrating how the static solution form can be redefined as a time inhomogeneous Markov chain. This approach offers thus a more realistic representation of repeated games. The last state affects the future state in two ways; directly via its probability distribution over states (status quo) but also by its impact on the players' preference order, allowing for endogenous preference changes. This impact can be flexibly modelled and easily adopted to a variety of repeated games. Its integration and complementation with other approaches should provide a valuable source for future research. 
A Tables 
Table 19. 2 groups - Rounded probabilities per Strategy profile

\begin{tabular}{|c|c|c|c|c|c|c|c|c|c|c|c|c|c|c|c|c|c|c|c|c|c|}
\hline \multicolumn{22}{|c|}{ From period 0 to 100 in steps of 5 periods } \\
\hline & 0 & 5 & 10 & 15 & 20 & 25 & 30 & 35 & 40 & 45 & 50 & 55 & 60 & 65 & 70 & 75 & 80 & 85 & 90 & 95 & 100 \\
\hline $\mathbf{0}$ & 1.00 & 0 & 0 & 0 & 0 & 0 & 0 & 0 & 0 & 0 & 0 & 0 & 0.01 & 0.02 & 0.07 & 0.18 & 0.37 & 0.6 & 0.81 & 0.94 & 1.00 \\
\hline 1 & 0 & 0 & 0 & 0 & 0 & 0 & 0 & 0 & 0 & 0 & 0 & 0 & 0 & 0 & 0.01 & 0.01 & 0.02 & 0.02 & 0.01 & 0 & 0 \\
\hline $\mathbf{3}$ & 0 & 0 & 0 & 0 & 0 & 0 & 0 & 0 & 0 & 0 & 0 & 0 & 0 & 0 & 0 & 0 & 0 & 0 & 0 & 0 & 0 \\
\hline 4 & 0 & 0 & 0 & 0 & 0 & 0 & 0 & 0 & 0 & 0 & 0 & 0 & 0 & 0 & 0 & 0 & 0 & 0 & 0 & 0 & 0 \\
\hline 5 & 0 & 0 & 0 & 0 & 0 & 0 & 0 & 0 & 0 & 0 & 0 & 0 & 0 & 0 & 0 & 0 & 0 & 0 & 0 & 0 & 0 \\
\hline 7 & 0 & 0.95 & 0.90 & 0.85 & 0.8 & 0.75 & 0.70 & 0.65 & 0.60 & 0.55 & 0.50 & 0.45 & 0.40 & 0.34 & 0.27 & 0.19 & 0.11 & 0.04 & 0.01 & 0 & 0 \\
\hline 12 & 0 & 0 & 0 & 0 & 0 & 0 & 0 & 0 & 0 & 0 & 0 & 0 & 0 & 0.01 & 0.03 & 0.06 & 0.09 & 0.11 & 0.09 & 0.05 & 0 \\
\hline 13 & 0 & 0 & 0 & 0 & 0 & 0 & 0 & 0 & 0 & 0 & 0 & 0 & 0 & 0 & 0 & 0 & 0 & 0 & 0 & 0 & 0 \\
\hline 15 & 0 & 0.05 & 0.10 & 0.15 & 0.20 & 0.25 & 0.30 & 0.35 & 0.40 & 0.45 & 0.50 & 0.55 & 0.59 & 0.62 & 0.62 & 0.55 & 0.41 & 0.23 & 0.08 & 0.01 & 0 \\
\hline
\end{tabular}


Table 20. Static game - 3 groups

\begin{tabular}{|c|c|c|c|c|c|c|c|c|c|c|c|c|c|c|c|}
\hline \multicolumn{16}{|c|}{ start-start-start } \\
\hline overall stability & $\mathrm{x}$ & $E$ & $\mathrm{x}$ & $\mathrm{x}$ & $E$ & $\overline{\mathrm{x}}$ & 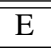 & $\overline{\mathrm{x}}$ & $\overline{\mathrm{x}}$ & $\overline{\mathrm{X}}$ & $\overline{\mathrm{x}}$ & $\overline{\mathrm{x}}$ & $\overline{\mathrm{x}}$ & $\overline{\mathrm{x}}$ & $\overline{\mathrm{X}}$ \\
\hline stability for $A$ & $\mathrm{r}$ & $\bar{r}$ & $\mathrm{r}$ & $\mathrm{r}$ & $\mathrm{r}$ & S & $\mathrm{S}$ & $\mathrm{r}$ & $\mathrm{r}$ & $\mathrm{S}$ & $\mathrm{u}$ & $\mathrm{r}$ & $\mathrm{r}$ & $\mathrm{u}$ & $\mathrm{u}$ \\
\hline$A$ 's preference order & 7 & 15 & 31 & 0 & 2 & 3 & 1 & 11 & 8 & 9 & 10 & 27 & 25 & 24 & 26 \\
\hline DPs & & & & & & 2 & 0 & & & 8 & 11 & & & 25 & 27 \\
\hline & & & & & & & & & & & & & & & \\
\hline stability for $C$ & $\mathrm{r}$ & $\mathrm{r}$ & $\mathrm{r}$ & $\mathrm{r}$ & $\mathrm{r}$ & $\mathrm{s}$ & $\mathrm{s}$ & $\mathrm{r}$ & $\mathrm{r}$ & $\mathrm{s}$ & $\mathrm{u}$ & $\mathrm{r}$ & $\mathrm{r}$ & $\mathrm{u}$ & $\mathrm{u}$ \\
\hline$C$ 's preference order & 7 & 15 & 31 & 0 & 1 & 3 & 2 & 11 & 8 & 10 & 9 & 27 & 26 & 24 & 25 \\
\hline DPs & & & & & & 1 & 0 & & & 8 & 11 & & & 26 & 27 \\
\hline & & & & & & & & & & & & & & & \\
\hline stability for $B$ & $\mathrm{r}$ & $\mathrm{u}$ & $\mathrm{u}$ & $\mathrm{u}$ & $\mathrm{u}$ & $\mathrm{u}$ & $\mathrm{r}$ & $\mathrm{r}$ & $\mathrm{s}$ & $\mathrm{s}$ & $\mathrm{s}$ & $\mathrm{s}$ & $\mathrm{r}$ & $\mathrm{u}$ & $\mathrm{u}$ \\
\hline$B$ 's preference order & 15 & 7 & 31 & 11 & 3 & 27 & $\overline{9}$ & $\overline{10}$ & $\overline{1}$ & $\overline{2}$ & $\overline{25}$ & 26 & 24 & 8 & 0 \\
\hline \multirow[t]{5}{*}{ DPs } & & 15 & 15 & 15 & 15 & 15 & & & 9 & 10 & 9 & 10 & & 24 & 24 \\
\hline & & & 7 & 7 & 7 & 7 & & & & & 1 & 2 & & & 8 \\
\hline & & & & 31 & 31 & 31 & & & & & & & & & \\
\hline & & & & & 11 & 11 & & & & & & & & & \\
\hline & & & & & & 3 & & & & & & & & & \\
\hline \multicolumn{16}{|c|}{ end-end-end } \\
\hline overall stability & $\bar{x}$ & $\overline{\mathrm{x}}$ & $\bar{E}$ & $\bar{E}$ & $\bar{x}$ & $\overline{\mathrm{X}}$ & $\overline{\mathrm{X}}$ & $\bar{x}$ & $\overline{\mathrm{x}}$ & $\bar{x}$ & $\bar{x}$ & $\bar{x}$ & $\bar{x}$ & $\overline{\mathrm{X}}$ & $\bar{x}$ \\
\hline stability for $A$ & $\mathrm{r}$ & $\mathrm{r}$ & $\mathrm{r}$ & $\mathrm{r}$ & $\mathrm{r}$ & $\mathrm{r}$ & $\mathrm{r}$ & $\mathrm{r}$ & $\mathrm{r}$ & $\mathrm{u}$ & $\mathrm{u}$ & $\mathrm{u}$ & $\mathrm{u}$ & $\mathrm{u}$ & $\mathrm{u}$ \\
\hline$A$ 's preference order & 7 & 15 & 31 & 0 & 8 & 24 & 2 & 10 & 26 & 1 & 9 & 25 & 3 & 11 & 27 \\
\hline DPs & & & & & & & & & & 0 & 8 & 24 & 2 & 10 & 26 \\
\hline & & & & & & & & & & & & & & & \\
\hline stability for $C$ & $\mathrm{r}$ & $\mathrm{r}$ & $\mathrm{r}$ & $\mathrm{r}$ & $\mathrm{r}$ & $\mathrm{r}$ & $\mathrm{r}$ & $\mathrm{r}$ & $\mathrm{r}$ & $\mathrm{u}$ & $\mathrm{u}$ & $\mathrm{u}$ & $\mathrm{u}$ & $\mathrm{u}$ & $\mathrm{u}$ \\
\hline$C$ 's preference order & 7 & 15 & 31 & 0 & 8 & 24 & 1 & 9 & 25 & 2 & 10 & 26 & 3 & 11 & 27 \\
\hline DPs & & & & & & & & & & 0 & 8 & 24 & 1 & 9 & 25 \\
\hline & & & & & & & & & & & & & & & \\
\hline stability for $B$ & $\mathrm{r}$ & $\mathrm{s}$ & $\mathrm{s}$ & $\mathrm{s}$ & $\mathrm{u}$ & $\mathrm{u}$ & $\mathrm{r}$ & $\mathrm{r}$ & $\mathrm{s}$ & $\mathrm{s}$ & $\mathrm{s}$ & $\mathrm{s}$ & $\mathrm{r}$ & $\mathrm{u}$ & $\mathrm{u}$ \\
\hline$B$ 's preference order & 27 & 11 & 3 & 31 & 15 & 7 & $\overline{9}$ & $\overline{10}$ & $\overline{1}$ & $\overline{2}$ & $\overline{25}$ & $\overline{26}$ & 0 & 8 & 24 \\
\hline \multirow[t]{5}{*}{ DPs } & & 27 & 27 & 27 & 27 & 27 & & & 9 & 10 & 9 & 10 & & 0 & 0 \\
\hline & & & 11 & 11 & 11 & 11 & & & & & 1 & 2 & & & 8 \\
\hline & & & & 3 & 3 & 3 & & & & & & & & & \\
\hline & & & & & 31 & 31 & & & & & & & & & \\
\hline & & & & & & 15 & & & & & & & & & \\
\hline
\end{tabular}


Table 21. Individual Transition Matrices - 3 groups

\begin{tabular}{|c|c|c|c|c|c|c|c|c|c|c|c|c|c|c|c|}
\hline \multicolumn{16}{|c|}{ Transition Matrix for $A$} \\
\hline & $\mathbf{0}$ & 1 & 2 & 3 & 7 & 8 & 9 & 10 & 11 & 15 & 24 & 25 & 26 & 27 & 31 \\
\hline 0 & 1 & $\alpha$ & 0 & 0 & 0 & 0 & 0 & 0 & 0 & 0 & 0 & 0 & 0 & 0 & 0 \\
\hline 1 & 0 & $1-\alpha$ & 0 & 0 & 0 & 0 & 0 & 0 & 0 & 0 & 0 & 0 & 0 & 0 & 0 \\
\hline 2 & 0 & 0 & 1 & $\alpha$ & 0 & 0 & 0 & 0 & 0 & 0 & 0 & 0 & 0 & 0 & 0 \\
\hline 3 & 0 & 0 & 0 & $1-\alpha$ & 0 & 0 & 0 & 0 & 0 & 0 & 0 & 0 & 0 & 0 & 0 \\
\hline 7 & 0 & 0 & 0 & 0 & 1 & 0 & 0 & 0 & 0 & 0 & 0 & 0 & 0 & 0 & 0 \\
\hline 8 & 0 & 0 & 0 & 0 & 0 & 1 & $\alpha$ & 0 & 0 & 0 & 0 & 0 & 0 & 0 & 0 \\
\hline 9 & 0 & 0 & 0 & 0 & 0 & $\frac{1}{0}$ & $\frac{\alpha}{1-\alpha}$ & 0 & 0 & 0 & 0 & 0 & 0 & 0 & 0 \\
\hline 10 & 0 & 0 & 0 & 0 & 0 & 0 & 0 & $\alpha$ & $\alpha$ & 0 & 0 & 0 & 0 & 0 & 0 \\
\hline 11 & 0 & 0 & 0 & 0 & 0 & 0 & 0 & $1-\alpha$ & $1-\alpha$ & 0 & 0 & 0 & 0 & 0 & 0 \\
\hline 15 & 0 & 0 & 0 & 0 & 0 & 0 & 0 & 0 & 0 & 1 & 0 & 0 & 0 & 0 & 0 \\
\hline 24 & 0 & 0 & 0 & 0 & 0 & 0 & 0 & 0 & 0 & 0 & $\alpha$ & $\alpha$ & 0 & 0 & 0 \\
\hline 25 & 0 & 0 & 0 & 0 & 0 & 0 & 0 & 0 & 0 & 0 & $\frac{\alpha}{1-\alpha}$ & $\frac{\omega}{1-\alpha}$ & 0 & 0 & 0 \\
\hline 26 & 0 & 0 & 0 & 0 & 0 & 0 & 0 & 0 & 0 & 0 & 0 & 0 & $\alpha$ & $\alpha$ & 0 \\
\hline 27 & 0 & 0 & 0 & 0 & 0 & 0 & 0 & 0 & 0 & 0 & 0 & 0 & $1-\alpha$ & $1-\alpha$ & 0 \\
\hline 31 & 0 & 0 & 0 & 0 & 0 & 0 & 0 & 0 & 0 & 0 & 0 & 0 & 0 & 0 & 1 \\
\hline \multicolumn{16}{|c|}{ Transition Matrix for $C$} \\
\hline & 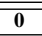 & 1 & $\overline{22}$ & 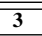 & 7 & 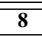 & 9 & 10 & $\overline{11}$ & $\overline{15}$ & 24 & 25 & 26 & 27 & 31 \\
\hline 0 & 1 & 0 & $\beta$ & 0 & 0 & 0 & 0 & 0 & 0 & 0 & 0 & 0 & 0 & 0 & 0 \\
\hline 1 & 0 & 1 & 0 & $\beta$ & 0 & 0 & 0 & 0 & 0 & 0 & 0 & 0 & 0 & 0 & 0 \\
\hline 2 & 0 & 0 & $1-\beta$ & 0 & 0 & 0 & 0 & 0 & 0 & 0 & 0 & 0 & 0 & 0 & 0 \\
\hline 3 & 0 & 0 & 0 & $1-\beta$ & 0 & 0 & 0 & 0 & 0 & 0 & 0 & 0 & 0 & 0 & 0 \\
\hline 7 & 0 & 0 & 0 & 0 & 1 & 0 & 0 & 0 & 0 & 0 & 0 & 0 & 0 & 0 & 0 \\
\hline 8 & 0 & 0 & 0 & 0 & 0 & 1 & 0 & $\beta$ & 0 & 0 & 0 & 0 & 0 & 0 & 0 \\
\hline 9 & 0 & 0 & 0 & 0 & 0 & 1 & $\beta$ & $\begin{array}{l}p \\
0\end{array}$ & $\beta$ & 0 & 0 & 0 & 0 & 0 & 0 \\
\hline 10 & 0 & 0 & 0 & 0 & 0 & 0 & 0 & $1-\beta$ & 0 & 0 & 0 & 0 & 0 & 0 & 0 \\
\hline 11 & 0 & 0 & 0 & 0 & 0 & 0 & $1-\beta$ & 0 & $1-\beta$ & 0 & 0 & 0 & 0 & 0 & 0 \\
\hline 15 & 0 & 0 & 0 & 0 & 0 & 0 & 0 & 0 & 0 & 1 & 0 & 0 & 0 & 0 & 0 \\
\hline 24 & 0 & 0 & 0 & 0 & 0 & 0 & 0 & 0 & 0 & 0 & $\beta$ & 0 & $\beta$ & 0 & 0 \\
\hline 25 & 0 & 0 & 0 & 0 & 0 & 0 & 0 & 0 & 0 & 0 & 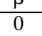 & $\beta$ & 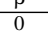 & $\beta$ & 0 \\
\hline 26 & 0 & 0 & 0 & 0 & 0 & 0 & 0 & 0 & 0 & 0 & $1-\beta$ & 0 & $1-\beta$ & 0 & 0 \\
\hline 27 & 0 & 0 & 0 & 0 & 0 & 0 & 0 & 0 & 0 & 0 & 0 & $1-\beta$ & 0 & $1-\beta$ & 0 \\
\hline 31 & 0 & 0 & 0 & 0 & 0 & 0 & 0 & 0 & 0 & 0 & 0 & 0 & 0 & 0 & 1 \\
\hline & & & & & & & & & & & & & & & \\
\hline \multicolumn{16}{|c|}{ Transition Matrix for $B$} \\
\hline & $\overline{00}$ & 1 & $\overline{22}$ & $\overline{3}$ & $\overline{7}$ & $\overline{88}$ & 9 & 10 & 11 & $\overline{15}$ & 24 & 25 & 26 & 27 & 31 \\
\hline 0 & $\gamma$ & 0 & 0 & 0 & 0 & $\gamma$ & 0 & 0 & 0 & 0 & $\gamma$ & 0 & 0 & 0 & 0 \\
\hline 1 & 0 & 1 & 0 & 0 & 0 & 0 & 0 & 0 & 0 & 0 & 0 & 0 & 0 & 0 & 0 \\
\hline 2 & 0 & 0 & 1 & 0 & 0 & 0 & 0 & 0 & 0 & 0 & 0 & 0 & 0 & 0 & 0 \\
\hline 3 & 0 & 0 & 0 & $\gamma$ & 0 & 0 & 0 & 0 & 0 & 0 & 0 & 0 & 0 & 0 & 0 \\
\hline 7 & 0 & 0 & 0 & 0 & 0 & 0 & 0 & 0 & 0 & 0 & 0 & 0 & 0 & 0 & 0 \\
\hline 8 & 0 & 0 & 0 & 0 & 0 & 0 & 0 & 0 & 0 & 0 & 0 & 0 & 0 & 0 & 0 \\
\hline 9 & 0 & 0 & 0 & 0 & 0 & 0 & 1 & 0 & 0 & 0 & 0 & 0 & 0 & 0 & 0 \\
\hline 10 & 0 & 0 & 0 & 0 & 0 & 0 & 0 & 1 & 0 & 0 & 0 & 0 & 0 & 0 & 0 \\
\hline 11 & 0 & 0 & 0 & 0 & 0 & 0 & 0 & 0 & $\gamma$ & 0 & 0 & 0 & 0 & 0 & 0 \\
\hline 15 & 0 & 0 & 0 & $1-\gamma$ & $1-\gamma$ & 0 & 0 & 0 & $1-\gamma$ & $1-\gamma$ & 0 & 0 & 0 & $1-\gamma$ & $1-\gamma$ \\
\hline 24 & $1-\gamma$ & 0 & 0 & $\frac{\gamma}{0}$ & $\frac{1 \gamma}{0}$ & $1-\gamma$ & 0 & 0 & $\frac{1}{1+\gamma}$ & $\frac{\gamma}{0}$ & $1-\gamma$ & 0 & 0 & 0 & $\frac{\gamma}{0}$ \\
\hline 25 & 0 & 0 & 0 & 0 & 0 & 0 & 0 & 0 & 0 & 0 & 0 & 1 & 0 & 0 & 0 \\
\hline 26 & 0 & 0 & 0 & 0 & 0 & 0 & 0 & 0 & 0 & 0 & 0 & 0 & 1 & 0 & 0 \\
\hline 27 & 0 & 0 & 0 & 0 & $\gamma$ & 0 & 0 & 0 & 0 & $\gamma$ & 0 & 0 & 0 & $\gamma$ & 0 \\
\hline 31 & 0 & 0 & 0 & 0 & 0 & 0 & 0 & 0 & 0 & 0 & 0 & 0 & 0 & 0 & $\gamma$ \\
\hline
\end{tabular}


Table 22. Game Transition Matrix

\begin{tabular}{|c|c|c|c|c|c|c|c|c|c|c|c|c|c|c|c|}
\hline \multicolumn{16}{|c|}{ Final Transition Matrix for the Game - 3 groups } \\
\hline 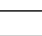 & 0 & 1 & 2 & 3 & 7 & 8 & 9 & 10 & 11 & 15 & 24 & 25 & 26 & 27 & 31 \\
\hline 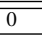 & $\overline{\gamma \gamma}$ & $\overline{\alpha \alpha}$ & $\overline{\bar{\beta}}$ & $\overline{\alpha \beta \gamma \gamma}$ & 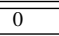 & $\overline{\gamma \gamma}$ & 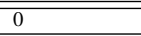 & 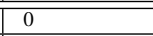 & $\overline{00}$ & 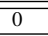 & $\overline{\alpha \beta \gamma \gamma}$ & 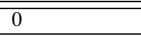 & 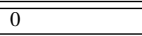 & 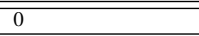 & 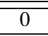 \\
\hline 1 & 0 & 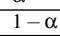 & 0 & $(1-\alpha) \beta \gamma$ & 0 & 0 & 0 & 0 & 0 & 0 & $(1-\alpha) \beta \gamma$ & 0 & 0 & 0 & 0 \\
\hline 2 & 0 & 0 & $1-\beta$ & $\alpha(1-\beta) \gamma$ & 0 & 0 & 0 & 0 & 0 & 0 & $\alpha(1-\beta) \gamma$ & 0 & 0 & 0 & 0 \\
\hline 3 & 0 & 0 & 0 & $(1-\alpha)(1-\beta) \gamma$ & 0 & 0 & 0 & 0 & 0 & 0 & $(1-\alpha)(1-\beta) \gamma$ & 0 & 0 & 0 & 0 \\
\hline 7 & 0 & 0 & 0 & 0 & 0 & 0 & 0 & 0 & 0 & 0 & 0 & 0 & 0 & 0 & 0 \\
\hline 8 & 0 & 0 & 0 & $\alpha \beta(1-\gamma)$ & 0 & 0 & $\alpha \beta$ & $\alpha \beta$ & $\alpha \beta$ & 0 & 0 & 0 & 0 & $\alpha \beta(1-\gamma)$ & 0 \\
\hline 9 & 0 & 0 & 0 & $(1-\alpha) \beta(1-\gamma)$ & 0 & 0 & $(1-\alpha) \beta$ & $(1-\alpha) \beta$ & $(1-\alpha) \beta$ & 0 & 0 & 0 & 0 & $(1-\alpha) \beta(1-\gamma)$ & 0 \\
\hline 10 & 0 & 0 & 0 & $\alpha(1-\beta)(1-\gamma)$ & 0 & 0 & $\alpha(1-\beta)$ & $\alpha(1-\beta)$ & $\alpha(1-\beta)$ & 0 & 0 & 0 & 0 & $\alpha(1-\beta)(1-\gamma)$ & 0 \\
\hline 11 & 0 & 0 & 0 & 0 & 0 & 0 & $(1-\alpha)(1-\beta)$ & $(1-\alpha)(1-\beta)$ & $(1-\alpha)(1-\beta) \gamma$ & 0 & 0 & 0 & 0 & 0 & 0 \\
\hline 15 & 0 & 0 & 0 & $(1-\alpha)(1-\beta)(1-\gamma)$ & $1-\gamma$ & 0 & 0 & 0 & $(1-\alpha)(1-\beta)(1-\gamma)$ & $1-\gamma$ & 0 & 0 & 0 & $(1-\alpha)(1-\beta)(1-\gamma)$ & $1-\gamma$ \\
\hline 24 & $1-\gamma$ & 0 & 0 & 0 & 0 & $1-\gamma$ & 0 & 0 & 0 & 0 & $\alpha \beta(1-\gamma)$ & $\alpha \beta$ & $\alpha \beta$ & $\alpha \beta \gamma$ & \begin{tabular}{|ll}
$1-7$ \\
0
\end{tabular} \\
\hline 25 & 0 & 0 & 0 & 0 & 0 & 0 & 0 & 0 & 0 & 0 & $(1-\alpha) \beta(1-\gamma)$ & $(1-\alpha) \beta$ & $(1-\alpha) \beta$ & $(1-\alpha) \beta \gamma$ & 0 \\
\hline 26 & 0 & 0 & 0 & 0 & 0 & 0 & 0 & 0 & 0 & 0 & $\alpha(1-\beta)(1-\gamma)$ & $\alpha(1-\beta)$ & $\alpha(1-\beta)$ & $\alpha(1-\beta) \gamma$ & 0 \\
\hline 27 & 0 & 0 & 0 & 0 & $\gamma$ & 0 & 0 & 0 & 0 & $\gamma$ & $(1-\alpha)(1-\beta)(1-\gamma)$ & $(1-\alpha)(1-\beta)$ & $(1-\alpha)(1-\beta)$ & $(1-\alpha)(1-\beta) \gamma$ & 0 \\
\hline 31 & 0 & 0 & 0 & 0 & 10 & 0 & 0 & 0 & 0 & 10 & 0 & 0 & 0 & 0 & $\gamma$ \\
\hline
\end{tabular}


Table 23. Transition Probabilities for simulation - 3 groups

\begin{tabular}{|c|c|c|c|c|c|c|c|c|c|c|c|c|c|c|c|c|c|c|c|c|c|c|c|c|c|c|c|c|c|c|c|c|}
\hline . & 25 & $\overline{50}$ & 75 & 10 & 125 & 150 & 1775 & 200 & 225 & 250 & 275 & 300 & 325 & 350 & 375 & 4400 & 425 & 450 & 4775 & \begin{tabular}{c|}
500 \\
\end{tabular} & 525 & 550 & 875 & 2600 & 625 & 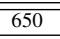 & 6765 & $\begin{array}{l}700 \\
\end{array}$ & 725 & $\begin{array}{l}750 \\
\end{array}$ & $\begin{array}{l}775 \\
\end{array}$ & 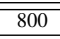 \\
\hline 0 & 0 & 0.01 & 0.02 & 0.03 & 0.02 & 0.02 & 0.01 & 0 & 0.01 & 0.02 & 0.02 & 0.02 & 0.02 & 0.01 & 0 & 0 & 0 & 0.01 & 0.02 & 0.03 & 0.02 & 0.01 & 0.01 & 0.01 & 0.01 & 0.02 & 0.03 & 0.03 & 0.02 & 0.01 & 0 & \\
\hline 1 & 0.02 & 0.01 & 0 & 0 & 0 & 0.01 & 0.02 & 0.03 & 0.02 & 0.01 & 0 & 0 & 0 & 0.01 & 0.02 & 0.02 & 0.02 & 0.01 & 0 & 0 & 0.01 & 0.01 & 0.02 & 0.02 & 0.02 & 0.01 & 0 & 0 & 0.01 & 0.01 & 0.02 & 0.02 \\
\hline 2 & 0 & 0 & 0 & 0 & 0 & 0 & 0 & 0 & 0 & 0 & 0 & 0 & 0 & 0 & 0 & 0 & 0 & 0 & 0 & 0 & 0 & 0 & 0 & 0 & 0 & 0 & 0 & 0 & 0 & 0 & 0 & 0 \\
\hline 3 & 0 & 0 & 0 & 0 & 0 & 0 & 0 & 0 & 0 & 0 & 0 & 0 & 0 & 0 & 0 & 0 & 0 & 0 & 0 & 0 & 0 & 0 & 0 & 0 & 0 & 0 & 0 & 0 & 0 & 0 & 0 & 0 \\
\hline 7 & 0 & 0 & 0 & 0 & 0 & 0 & 0 & 0 & 0 & 0 & 0 & 0 & 0 & 0 & 0 & 0 & 0 & 0 & 0 & 0 & 0 & 0 & 0 & 0 & 0 & 0 & 0 & 0 & 0 & 0 & 0 & 0 \\
\hline 8 & 0 & 0.04 & 0.13 & 0.23 & 0.24 & 0.15 & 0.04 & 0 & 0.03 & 0.04 & 0.01 & 0 & 0.02 & 0.04 & 0.02 & 0 & 0.06 & 0.18 & 0.25 & 0.21 & 0.1 & 0.02 & 0 & 0 & 0.01 & 0.06 & 0.17 & 0.25 & 0.22 & 0.11 & 0.02 & 0 \\
\hline 9 & 0.02 & 0.04 & 0.02 & 0 & 0.05 & 0.16 & 0.25 & 0.22 & 0.12 & 0.03 & 0 & 0 & 0 & 0.05 & 0.15 & 0.24 & 0.24 & 0.13 & 0.03 & 0 & 0.03 & 0.04 & 0.01 & 0 & 0.03 & 0.04 & 0.01 & & 0.08 & 0.2 & 0.26 & 0.2 \\
\hline 10 & 0.04 & 0.12 & 0.13 & 0.08 & 0.02 & 0 & 0 & 0 & 0.03 & 0.13 & 0.26 & 0.31 & 0.23 & 0.1 & 0.01 & 0 & 0 & 0 & 0.03 & 0.09 & 0.13 & 0.1 & 0.02 & 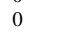 & 0.07 & 0.13 & 0.12 & 0.05 & 0.01 & 0 & 0.20 & 0 \\
\hline 11 & 0.18 & 0.09 & 0.02 & 0 & 0 & 0 & 0.02 & 0.08 & 0.13 & 0.1 & 0.03 & 0 & 0.04 & 0.09 & 0.09 & 0.05 & 0.01 & 0 & 0 & 0 & 0.04 & 0.14 & 0.27 & 0.3 & 0.2 & 0.07 & 0.01 & 0 & 0 & 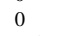 & 0.03 & 0.07 \\
\hline 15 & 0.7 & 0.59 & 0.46 & 0.33 & 0.2 & 0.09 & 0.02 & 0 & 0.03 & 0.11 & 0.22 & 0.34 & 0.48 & 0.6 & 0.67 & 0.67 & 0.62 & 0.54 & 0.43 & 0.3 & 0.18 & 0.08 & 0.02 & 0 & 0.04 & 0.13 & 0.24 & 0.37 & 0.49 & 0.59 & 0.65 & 0.68 \\
\hline 24 & 0 & 0.01 & 0.01 & 0.01 & 0.01 & 0.01 & 0 & 0 & 0 & 0 & 0 & 0 & 0 & 0.01 & 0.01 & 0 & 0.01 & 0.01 & 0.01 & 0.01 & 0.01 & 0 & 0 & 0 & 0 & 0 & 0.01 & 0.01 & 0.01 & 0.01 & 0.01 & 0 \\
\hline 25 & 0 & 0 & 0 & 0 & 0 & 0.01 & 0.01 & 0.01 & 0 & 0 & 0 & 0 & 0 & 0 & 0.01 & 0.01 & 0.01 & 0 & 0 & 0 & 0 & 0 & 0 & 0 & 0 & 0 & 0 & 0 & 0 & 0.01 & 0.01 & 0.01 \\
\hline 26 & 0 & 0 & 0 & 0 & 0 & 0 & 0 & 0 & 0 & 0 & 0.01 & 0.01 & 0.01 & 0 & 0 & 0 & 0 & 0 & 0 & 0 & 0.01 & 0 & 0 & 0 & 0 & 0.01 & 0 & 0 & 0 & 0 & 0 & 0 \\
\hline 27 & 0.03 & 0.1 & 0.2 & 0.33 & 0.45 & 0.56 & 0.63 & 0.65 & 0.63 & 0.55 & 0.44 & 0.31 & 0.19 & 0.09 & 0.02 & 0 & 0.03 & 0.11 & 0.23 & 0.35 & 0.48 & 0.58 & 0.65 & 0.66 & 0.62 & 0.53 & 0.41 & 0.29 & 0.16 & 0.07 & 0.01 & 0.01 \\
\hline 31 & 0 & 0 & 0 & 0 & 0 & 0 & 0 & 0 & 0 & 0 & 0 & 0 & 0 & 0 & 0 & 0 & 0 & 0 & 0 & 0 & 0 & 0 & 0 & 0 & 0 & 0 & 0 & 0 & 0 & 0 & 0 & 0 \\
\hline
\end{tabular}




\section{B References}

\section{References}

Dosi, G., Fagiolo, G., Aversi, R., Meacci, M., and Olivetti, C. (1999). Cognitive Processes, Social Adaptation and Innovation in Consumption Patterns: from Stylized Facts to Demand Theory. Edward Elgar.

Dosi, G., Hobday, M., and Marengo, L. (2003). Problem-solving Behaviors, Organizational Forms, and the Complexity of Tasks. Blackwell Publishing.

Fagiolo, G. and Dosi, G. (2003). Exploitation, exploration and innovation in a model of endogenous growth with locally interacting agents. Structural change and Economic Dynamics, 14(2002/25).

Fraser, N. M. and Hipel, K. W. (1984). Conflict Analysis: Models and Resolutions. Elsevier Science Ltd.

Ille, S. (2012). The theory of conflict analysis: A review of the approach by keith w. hipel \& niall m. fraser. submitted to: International Journal of Mathematics, Game Theory and Algebra, under review.

Potts, J. (2000). The New Evolutionary Microeconomics: Complexity, Competence, and Adaptive Behaviour. Edward Elgar Publishing Ltd. 